\title{
THE CONJECTURAL CONNECTIONS BETWEEN AUTOMORPHIC REPRESENTATIONS AND GALOIS REPRESENTATIONS
}

\author{
KEVIN BUZZARD AND TOBY GEE
}

\begin{abstract}
We state conjectures on the relationships between automorphic representations and Galois representations, and give evidence for them.
\end{abstract}

\section{Contents}

1. Introduction.

2. $\quad L$-groups and local definitions.

3. Global definitions, and the first conjectures. 16

4. The case of tori. 22

5. Twisting and Gross' $\eta . \quad 27$

6. Functoriality. 34

7. Reality checks. 35

8. Relationship with theorems/conjectures in the literature. 37

References

\section{INTRODUCTION.}

1.1. Given an algebraic Hecke character for a number field $F$, a classical construction of Weil produces a compatible system of 1-dimensional $\ell$-adic representations of $\operatorname{Gal}(\bar{F} / F)$. In the late 1950s, Taniyama's work [Tan57] on $L$-functions of abelian varieties with complex multiplications led him to consider certain higherdimensional compatible systems of Galois representations, and by the 1960s it was realised by Serre and others that Weil's construction might well be the tip of a very large iceberg. Serre conjectured the existence of 2 -dimensional $\ell$-adic representations of $\operatorname{Gal}(\overline{\mathbb{Q}} / \mathbb{Q})$ attached to classical modular eigenforms for the group $\mathrm{GL}_{2}$ over $\mathbb{Q}$, and their existence was established by Deligne not long afterwards. Moreover, Langlands observed that one way to attack Artin's conjecture on the analytic continuation of Artin $L$-functions might be via first proving that any non-trivial $n$-dimensional irreducible complex representation of the absolute Galois group of a number field $F$ came (in some precise sense) from an automorphic representation for $\mathrm{GL}_{n} / F$, and then analytically continuing the $L$-function of this automorphic representation instead.

One might ask whether one can associate "Galois representations" to automorphic representations for an arbitrary connected reductive group over a number field. There are several approaches to formalising this problem. Firstly one could insist

2000 Mathematics Subject Classification. 11F33. 
on working with all automorphic representations and attempt to associate to them complex representations of a "Langlands group", a group whose existence is only conjectural but which, if it exists, should be much bigger than the absolute Galois group of the number field (and even much bigger than the Weil group of the number field) - a nice reference for a rigorous formulation of a conjecture here is Art02. Alternatively one could restrict to automorphic representations that are "algebraic" in some reasonable sense, and in this case one might attempt to associate certain complex representations of the fundamental group of some Tannakian category of motives, a group which might either be a pro-algebraic group scheme or a topological group. Finally, following the original examples of Weil and Deligne, one might again restrict to algebraic automorphic representations, and then attempt to associate compatible systems of $\ell$-adic Galois representations to such objects (that is, representations of the absolute Galois group of the number field over which the group is defined). The advantage of the latter approach is that it is surely the most concrete.

For the group $\mathrm{GL}_{n}$ over a number field, Clozel gave a definition of what it meant for an automorphic representation to be "algebraic". The definition was, perhaps surprisingly, a non-trivial twist of a notion which presumably had been in the air for many years. Clozel made some conjectures predicting that algebraic automorphic representations should give rise to $n$-dimensional $\ell$-adic Galois representations (so his conjecture encapsulates Weil's result on Hecke characters and Deligne's theorem too). Clozel proved some cases of his conjecture, when he could switch to a unitary group and use algebraic geometry to construct the representations.

The goal of this paper is to generalise (most of) the statement of Clozel's conjecture to the case where $\mathrm{GL}_{n}$ is replaced by an arbitrary connected reductive group $G$. Let us explain the first stumbling block in this programme. The naive conjecture would be of the following form: if an automorphic representation $\pi$ for $G$ is algebraic (in some reasonable sense) then there should be a Galois representation into the $\overline{\mathbb{Q}}_{p}$-points of the $L$-group of $G$, associated to $\pi$. But if one looks, for example, at Proposition 3.4.4 of [CHT08, one sees that they can associate $p$-adic Galois representations to certain automorphic representations on certain compact unitary groups, but that the Galois representations are taking values in a group $\mathcal{G}_{n}$ which one can check is not the $L$-group of the unitary group in question (for dimension reasons, for example). In fact there are even easier examples of this phenomenon: if $\pi$ is the automorphic representation for $\mathrm{GL}_{2} / \mathbb{Q}$ attached to an elliptic curve over the rationals, then (if one uses the standard normalisation for $\pi$ ) one sees that $\pi$ has trivial central character and hence descends to an automorphic representation for $\mathrm{PGL}_{2} / \mathbb{Q}$ which one would surely hope to be algebraic (because it is cohomological). However, the $L$-group of $\mathrm{PGL}_{2} / \mathbb{Q}$ is $\mathrm{SL}_{2}$ and there is no way of twisting the Galois representation afforded by the $p$-adic Tate module of the curve so that it lands into $\mathrm{SL}_{2}\left(\overline{\mathbb{Q}}_{\ell}\right)$, because the cyclotomic character has no square root (consider complex conjugation). On the other hand, there do exist automorphic representation for $\mathrm{PGL}_{2} / \mathbb{Q}$ which have associated Galois representations into $\mathrm{SL}_{2}\left(\overline{\mathbb{Q}}_{\ell}\right)$; for example one can easily build them from automorphic representations on $\mathrm{GL}_{2} / \mathbb{Q}$ constructed via the Langlands-Tunnell theorem applied to a continuous even irreducible 2-dimensional representation of $\mathrm{Gal}(\overline{\mathbb{Q}} / \mathbb{Q})$ into $\mathrm{SL}_{2}(\mathbb{C})$ with solvable image. What is going on? 
Our proposed solution is the following. For a general connected reductive group $G$, we believe that there are two reasonable notions of "algebraic". For $\mathrm{GL}_{n}$ these notions differ by a twist (and this explains why this twist appears in Clozel's work). For some groups the notions coincide. But for some others - for example $\mathrm{PGL}_{2}$ the notions are disjoint. The two definitions "differ by half the sum of the positive roots". We call the two notions $C$-algebraic and $L$-algebraic. It turns out that cohomological automorphic representations are $C$-algebraic (hence the $C$ ), and that given an $L$-algebraic automorphic representation one might expect an associated Galois representation into the $L$-group (hence the $L$ ). Clozel twists $C$-algebraic representations into $L$-algebraic ones in his paper, and hence conjectures that there should be Galois representations attached to $C$-algebraic representations for $\mathrm{GL}_{n}$, but this trick is not possible in general. In this paper we explicitly conjecture the existence of $p$-adic Galois representations associated to $L$-algebraic automorphic representations for a general connected reductive group over a number field.

On the other hand, one must not leave $C$-algebraic representations behind. For example, for certain unitary groups of even rank over the rationals, all automorphic representations are $C$-algebraic and none are $L$-algebraic at all! It would be a shame to have no conjecture at all in these cases. We show in section 5 that given a $C$ algebraic automorphic representation for a group $G$, it can be lifted to a $C$-algebraic representation for a certain covering group $\widetilde{G}$ (a canonical central extension of $G$ by $\left.G L_{1}\right)$ where there is enough space to twist $C$-algebraic representations into $L$ algebraic ones. After making such a twisting one would then conjecturally expect an associated Galois representation into the $L$-group not of $G$ but of $\widetilde{G}$. We define the $C$-group ${ }^{C} G$ of $G$ to be ${ }^{L} \widetilde{G}$. For example, if $\pi$ is the automorphic representation for the group $\mathrm{PGL}_{2} / \mathbb{Q}$ attached to an elliptic curve over $\mathbb{Q}$, we can verify that $\pi$ is $C$-algebraic, and that $\widetilde{G}=\mathrm{GL}_{2} / \mathbb{Q}$ in this case, and hence one would expect a Galois representation into $\mathrm{GL}_{2}\left(\overline{\mathbb{Q}}_{\ell}\right)$ associated to $\pi$, which is given by the Tate module of the curve. We also verify the compatibility of the construction with that made by Clozel-Harris-Taylor in the unitary group case.

In this paper, we explain the phenomena above in more detail. In particular we formulate a conjecture associating $p$-adic Galois representations to $L$-algebraic automorphic representations for an arbitrary connected reductive group over a number field, which appears to essentially include all known theorems and conjectures of this form currently in the literature. We initially imagined that such a conjecture was already "known to the experts". However, our experience has been that this is not the case; in fact, it seems that the issues that arise when comparing the definitions of $L$-algebraic and $C$-algebraic representations were a known problem, with no clear solution (earlier attempts to deal with this issue have been by means of redefining the local Langlands correspondence and the Satake isomorphism via a twist, as in [Gro99]; however this trick only works for certain groups). In one interesting example in the literature where Galois representations are attached to certain cohomological automorphic representations - the constructions of CHT08 - the Galois representations take values in a group $\mathcal{G}_{n}$, whose construction seemed to us to be one whose main motivation was that it was the group that worked, rather than the group that came from a more conceptual argument. We revisit this construction in 8.3 Ultimately, we hope that this article will clarify once and for all a variety of issues that occur when leaving the relative safety of $\mathrm{GL}_{n}$, giving a firm framework for further research on Galois representations into groups other than $\mathrm{GL}_{n}$. 
1.2. Acknowledgements. We would like to thank Jeff Adams, James Arthur, Frank Calegari, Brian Conrad, Matthew Emerton, Wee Teck Gan, Dick Gross, Florian Herzig, Robert Langlands, David Loeffler, Ambrus Pál, Richard Taylor and David Vogan for helpful discussions relating to this work. Particular thanks go to Gross, for giving us a copy of Deligne's 2007 letter to Serre and urging us to read it, and to Adams and Vogan for dealing with several questions of ours involving local Langlands at infinity which were apparently "known to the experts" but which we could not extract from the literature ourselves.

The first author was supported by an EPSRC Advanced Research Fellowship, and the second author would like to acknowledge the support of the National Science Foundation (award number DMS-0841491). He would also like to thank the mathematics department of Northwestern University for its hospitality in the early stages of this project.

\section{2. $L$-GROUPS AND LOCAL DEFINITIONS.}

In this section we give an overview of various standard facts concerning $L$-groups, the Satake isomorphism, the archimedean local Langlands correspondence, and basic Hodge-Tate theory, often with a specific emphasis on certain arithmetic aspects that are not considered relevant in many of the standard references. In summary: our $L$-groups will be over $\overline{\mathbb{Q}}$, we will keep track of the two different $\mathbb{Q}$-structures in the Satake isomorphism, and our local Langlands correspondence will concern representations of $G(\mathbb{R})$ or $G(\overline{\mathbb{R}})$, where $\overline{\mathbb{R}}$ is an algebraic closure of the reals which we do not identify with $\mathbb{C}$ (note that on the other hand, all our representations will be on $\mathbb{C}$-vector spaces). This section is relatively elementary but contains all of the crucial local definitions.

2.1. The $L$-group. We briefly review the notion of an $L$-group. We want to view the $L$-group of a connected reductive group as a group over $\overline{\mathbb{Q}}$, rather than the more traditional $\mathbb{C}$, as we shall later on be considering representations into the $\overline{\mathbb{Q}}_{p}$-points of the $L$-group. We review the standard definitions from the point of view that we shall be taking.

We take the approach to dual groups explained in section 1 of [Kot84], but work over $\overline{\mathbb{Q}}$. See also section 3.3 of Exposé XXIV of [ABD $\left.{ }^{+} 66\right]$, which is perhaps where the trick of taking limits of based root data is first introduced.

Let $k$ be a field and let $G$ be a connected reductive algebraic group over $k$. Fix once and for all a separable closure $k^{\text {sep }}$ of $k$, and let $\Gamma_{k}$ denote $\operatorname{Gal}\left(k^{\mathrm{sep}} / k\right)$. The group $G$ splits over $k^{\text {sep }}$, and if we choose a maximal torus $T$ in $G_{k^{\text {sep }}}$ and a Borel subgroup $B$ of $G_{k^{\text {sep }}}$ containing $T$, one can associate the based root datum $\Psi_{0}(G, B, T):=\left(X^{*}(T), \Delta^{*}(B), X_{*}(T), \Delta_{*}(B)\right)$ consisting of the character and cocharacter groups of $T$, and the roots and coroots which are simple and positive with respect to the ordering defined by $B$. Now let $Z_{G}$ denote the centre of $G$; then $\left(G / Z_{G}\right)\left(k^{\mathrm{sep}}\right)$ acts on $G_{k^{\text {sep }}}$ by conjugation, and if $T^{\prime}$ and $B^{\prime}$ are another choice of maximal torus and Borel then there is an element of $\left(G / Z_{G}\right)\left(k^{\mathrm{sep}}\right)$ sending $B^{\prime}$ to $B$ and $T^{\prime}$ to $T$, and all such elements induce the same isomorphisms of based root data $\Psi_{0}(G, B, T) \rightarrow \Psi_{0}\left(G, B^{\prime}, T^{\prime}\right)$. Following Kottwitz, we define $\Psi_{0}(G):=\left(X^{*}, \Delta^{*}, X_{*}, \Delta_{*}\right)$ to be the projective limit of the $\Psi_{0}(G, B, T)$ via these isomorphisms. This means in practice that given a maximal torus $T$ of $G_{k^{\text {sep }}}$, the group $X^{*}$ is isomorphic to the character group of $T$ but not canonically; however given also a Borel $B$ containing the torus, there is now a canonical map 
$X^{*}=X^{*}(T)$ (and different Borels give different canonical isomorphisms). There is a natural group homomorphism $\mu_{G}: \Gamma_{k} \rightarrow \operatorname{Aut}\left(\Psi_{0}(G)\right.$ ) (defined for example in $\S 1.3$ of [Bor79], or more conceptually by transport de structure) and if $K \subseteq k^{\text {sep }}$ is a Galois extension of $k$ that splits $G$ then $\mu_{G}$ factors through $\operatorname{Gal}(K / k)$.

We let $\widehat{G}$ denote a connected reductive group over $\overline{\mathbb{Q}}$ equipped with a given isomorphism $\Psi_{0}(\widehat{G})=\Psi_{0}(G)^{\vee}$, the dual root datum to $\Psi_{0}(G)$. There is a canonical group isomorphism $\operatorname{Aut}\left(\Psi_{0}(G)\right)=\operatorname{Aut}\left(\Psi_{0}(G)^{\vee}\right)$, sending an automorphism of $X^{*}$ to its inverse (one needs to insert this inverse to 'cancel out' the contravariance coming from the dual construction), and hence a canonical action of $\Gamma_{k}$ on $\Psi_{0}(G)^{\vee}$. If we choose a Borel, a maximal torus, and a splitting (also called a pinning; see p.10 of [Spr79 for details and definitions) of $\widehat{G}$ then, as on p.10 of [Spr79], this data induces a lifting $\operatorname{Aut}\left(\Psi_{0}(G)^{\vee}\right) \rightarrow \operatorname{Aut}(\widehat{G})$ and hence (via $\mu_{G}$ ) a left action of $\Gamma_{k}$ on $\widehat{G}$. We define the $L$-group ${ }^{L} G$ of $G$ to be the resulting semidirect product, regarded as a group scheme over $\overline{\mathbb{Q}}$ with identity component $\widehat{G}$ and component group $\Gamma_{k}$. For $K$ a field containing $\overline{\mathbb{Q}}$ we have ${ }^{L} G(K)=\widehat{G}(K) \rtimes \Gamma_{k}$. Often in the literature people use ${ }^{L} G$ to be the group that we call ${ }^{L} G(\mathbb{C})$.

Note that there is a fair amount of "ambiguity" in this definition. The group $\widehat{G}$ is "only defined up to inner automorphisms", as is the lifting of $\mu_{G}$. So, even if we fix our choice of $k^{\mathrm{sep}}$, points in ${ }^{L} G(K)$ are "only defined up to conjugation by $\widehat{G}(K)$ ".

Now say $F$ is a number field and $k=F_{v}$ is a completion of $F$ at a place $v$. If $G / F$ is a connected reductive group and $G_{v} / F_{v}$ is its base extension, then we would like to be able to relate the $L$-groups of $G$ and $G_{v}$ (after choosing algebraic closures $\bar{F}$ and $\bar{F}_{v}$ of $F$ and $F_{v}$ ). To do this it we choose a map $\bar{F} \rightarrow \bar{F}_{v}$; doing this gives us a way of pulling back our global choices of Borel and torus to the local group, and hence gives us a way of identifying $\Psi_{0}(G)$ and $\Psi_{0}\left(G_{v}\right)$ and of embedding the absolute Galois group of $F_{v}$ into that of $F$ in a manner compatible with the actions of these groups on the based root data. We thus get a natural map of algebraic groups from $L G_{v}$ to $L G$.

If $K$ is an extension of $\overline{\mathbb{Q}}$ and $\rho$ is a group homomorphism $\Gamma_{k} \rightarrow{ }^{L} G(K)$, then we say that $\rho$ is admissible if the map $\Gamma_{k} \rightarrow \Gamma_{k}$ induced by $\rho$ and the surjection ${ }^{L} G(K) \rightarrow \Gamma_{k}$ is the identity. We say two admissible $\rho$ s are equivalent if they differ by conjugation by an element of $\widehat{G}(K)$.

We remark that readers for whom even the choice of $k^{\text {sep }}$ is distasteful can avoid making this choice all together by interpreting $\widehat{G}(K)$ as a scheme of groups over $\operatorname{Spec}(k)$ and then interpreting equivalence classes of admissible $\rho$ s as above as elements of $H^{1}(\operatorname{Spec}(k), \widehat{G}(K))$.

We fix once and for all an embedding $\overline{\mathbb{Q}} \rightarrow \mathbb{C}$. Later on, when talking about Galois representations, we shall fix a prime number $p$ and an embedding $\overline{\mathbb{Q}} \rightarrow \overline{\mathbb{Q}}_{p}$. This will enable us to talk about the groups ${ }^{L} G(\mathbb{C})$ and ${ }^{L} G\left(\overline{\mathbb{Q}}_{p}\right)$.

2.2. Satake parameters. In this section, $k$ is a non-archimedean local field with integers $\mathcal{O}$, and we again fix a separable closure $k^{\text {sep }}$ of $k$ and set $\Gamma_{k}=\operatorname{Gal}\left(k^{\mathrm{sep}} / k\right)$. We normalise the reciprocity map $k^{\times} \rightarrow \Gamma_{k}^{\mathrm{ab}}$ of local class field theory so that it takes a uniformiser to a geometric Frobenius. We follow Tate's definitions and conventions for Weil groups - in brief, a Weil group $W_{k}=W_{k^{\text {sep }} / k}$ for $k$ comes equipped with maps $W_{k} \rightarrow \Gamma_{k}$ and $k^{\times} \rightarrow W_{k}^{\text {ab }}$ such that the induced map $k^{\times} \rightarrow \Gamma_{k}^{\mathrm{ab}}$ is the reciprocity homomorphism of class field theory, normalised as above. 
Let $G / k$ be connected reductive group which is furthermore unramified (that is, quasi-split, and split over an unramified extension of $k$ ). Then $G(k)$ has hyperspecial maximal compact subgroups (namely $\mathcal{G}(\mathcal{O}) \subseteq G(k)$, where $\mathcal{G}$ is any reductive group over $\mathcal{O}$ with generic fibre $G$ ); fix one, and call it $K$. Nothing we do will depend on this choice, but we will occasionally need to justify this. Let $B=B_{k}$ be a Borel in $G$ defined over $k$, let $T=T_{k}$ be a maximal torus of $B$, also defined over $k$, and let $T_{d}$ be the maximal $k$-split sub- $k$-torus of $T$. Let $W_{d}$ be the subgroup of the Weyl group of $G$ consisting of elements which map $T_{d}$ to itself. Let ${ }^{\circ} T$ denote the maximal compact subgroup of $T(k)$. It follows from an easy cohomological calculation (done for example in $\S 9.5$ of Bor79]) that the inclusion $T_{d} \rightarrow T$ induces an isomorphism of groups $T_{d}(k) / T_{d}(\mathcal{O}) \rightarrow T(k) /{ }^{\circ} T$. We normalise Haar measure on $G(k)$ so that $K$ has measure 1 (and remark that by 3.8.2 of Tit79] this normalisation is independent of the choice of hyperspecial maximal compact $K$ ). If $R$ is a field of characteristic zero then let $H_{R}(G(k), K)$ denote the Hecke algebra of bi- $K$-invariant $R$-valued functions on $G(k)$ with compact support, and with multiplication given by convolution. Similarly let $H_{R}\left(T(k),{ }^{o} T\right)$ denote the analogous Hecke algebra for $T(k)$ (with Haar measure normalised so that ${ }^{\circ} T$ has measure 1 ).

The Satake isomorphism (see for example $\S 4.2$ of Car79 ) is a canonical isomorphism $H_{\mathbb{C}}(G(k), K)=\mathbb{C}\left[X_{*}\left(T_{d}\right)\right]^{W_{d}}=H_{\mathbb{C}}\left(T_{d}(k), T_{d}(\mathcal{O})\right)^{W_{d}}=H_{\mathbb{C}}\left(T(k),{ }^{o} T\right)^{W_{d}}$, where $X_{*}\left(T_{d}\right)$ is the cocharacter group of $T_{d}$ (the Satake isomorphism is the first of these equalities; the others are easy). We normalise the Satake isomorphism in the usual way, so that it does not depend on the choice of the Borel subgroup containing $T$; this is the only canonical way to do things. This standard normalisation is however not in general "defined over $\mathbb{Q}$ "-for example if $k=\mathbb{Q}_{p}$ and $G=\mathrm{GL}_{2}$ and $K=\mathrm{GL}_{2}\left(\mathbb{Z}_{p}\right)$ then the Satake isomorphism sends the characteristic function of $K\left(\begin{array}{ll}p & 0 \\ 0 & 1\end{array}\right) K$ to a function on $T\left(\mathbb{Q}_{p}\right)$ taking the value $\sqrt{p}$ on the matrix $\left(\begin{array}{ll}p & 0 \\ 0 & 1\end{array}\right)$. This square root of $p$ appears because the definition of the Satake isomorphism involves a twist by half the sum of the positive roots of $G$ (see formula (19) of section 4.2 of [Car79]) and because of this twist, the isomorphism does not in general induce a canonical isomorphism $H_{\mathbb{Q}}(G(k), K)=\mathbb{Q}\left[X_{*}\left(T_{d}\right)\right]^{W_{d}}$.

In Gro99] and [Clo90 this issue of square roots is avoided by renormalising the Satake isomorphism. Let us stress that we shall not do this here, and we shall think of $H_{\mathbb{Q}}(G(k), K)$ and $\mathbb{Q}\left[X_{*}\left(T_{d}\right)\right]^{W_{d}}$ as giving two possibly distinct $\mathbb{Q}$-structures on the complex algebraic variety $\operatorname{Spec}\left(H_{\mathbb{C}}(G(k), K)\right)$ which shall perform two different functions - they will give us two (typically distinct) notions of being defined over a subfield of $\mathbb{C}$. We note however that if half the sum of the positive roots of $G$ is in the weight lattice $X^{*}(T)$ (this occurs for example if $G$ is semi-simple and simply connected, or a torus) then the map $\delta^{1 / 2}: T(k) \rightarrow \mathbb{R}_{>0}$ mentioned in formula (19) of Car79 is $\mathbb{Q}$-valued (see formula (4) of Car79] for the definition of $\delta$ ) and the proof of Theorem 4.1 of Car79 makes it clear that in this case the Satake isomorphism does induce an isomorphism $H_{\mathbb{Q}}(G(k), K)=\mathbb{Q}\left[X_{*}\left(T_{d}\right)\right]^{W_{d}}$.

Next we recall how the Satake isomorphism above leads us to an unramified local Langlands correspondence. Say $\pi$ is a smooth admissible irreducible complex representation of $G(k)$, and assume that $\pi^{K} \neq 0$ for our given choice of $K$ (so in particular, $\pi$ is unramified). Then $\pi^{K}$ is a 1-dimensional representation of $H_{\mathbb{C}}(G, K)$ and hence gives rise to a $\mathbb{C}$-valued character of $H_{\mathbb{C}}(G, K)$. Now results of Gantmacher and Langlands in section 6 of Bor79 enable one to canonically associate to $\pi$ an unramified continuous admissible representation $r_{\pi}: W_{k} \rightarrow{ }^{L} G(\mathbb{C})$, where 
"admissible" in this context means a group homomorphism such that if one composes it with the canonical map ${ }^{L} G(\mathbb{C}) \rightarrow \Gamma_{k}$ then one obtains the canonical map $W_{k} \rightarrow \Gamma_{k}$ (part of the definition of the Weil group) and "unramified" means that the resulting 1-cocycle $W_{k} \rightarrow \widehat{G}(\mathbb{C})$ is trivial on the inertia subgroup of $W_{k}$. In fact there are two ways of normalising the construction: if we follow section 6 of [Bor79] then in the crucial Proposition 6.7 Borel has chosen the $\sigma$ that appears there to be an arbitrary generator of the Galois group of a finite unramified extension of $k$ which splits $G$, and we are free to choose $\sigma$ to be either an arithmetic or a geometric Frobenius. We shall let $\sigma$ denote a geometric Frobenius: now an easy check (unravelling the definitions in Bor79 and Car79) shows that if $G=\mathrm{GL}_{1}$ and $\pi$ is an unramified representation of $\mathrm{GL}_{1}(k)$ then the corresponding Galois representation $W_{k} \rightarrow \mathrm{GL}_{1}(\mathbb{C})$ is the one induced by our given isomorphism $k^{\times}=W_{k}^{\mathrm{ab}}$. See Remark 3.2 .5 for some comments about what would have happened had we chosen an arithmetic Frobenius here, and normalised our class field theory isomorphisms so that they sent arithmetic Frobenii to uniformisers.

Remark 2.2.1. The local Langlands correspondence behaves in a natural way under certain unramified twists. Let $\xi: G \rightarrow \mathbb{G}_{m}$ be a character of $G$ defined over $k$. Then $\xi(K)$ is a compact subset of $k^{\times}$and is hence contained in $\mathcal{O}^{\times}$. If $s \in \mathbb{C}$ then we can define $\chi: G(k) \rightarrow \mathbb{C}^{\times}$by $\chi(g)=|\xi(g)|^{s}$; then $\chi(K)=1$. Hence for an irreducible $\pi$ with a $K$-fixed vector, $\pi \otimes \chi$ is also irreducible with a $K$-fixed vector. The map $\chi$ induces an automorphism of $H_{\mathbb{C}}(G(k), K)$ sending a function $f$ to the function $g \mapsto \chi(g) f(g)$, and similarly it induces automorphisms of $H_{\mathbb{C}}\left(T(k) /{ }^{o} T\right)$ and $H_{\mathbb{C}}\left(T_{d}(k) / T_{d}(\mathcal{O})\right)$. These latter two automorphisms are equivariant for the action of $W_{d}$, because $\chi$ is constant on $G(k)$-conjugacy classes. Furthermore, the Satake isomorphism commutes with these automorphisms - this follows without too much trouble from the formula defining the Satake isomorphism in $\S 4.2$ of Car79] and the observation that the kernel of $\xi$ contains the unipotent radical of any Borel in $G$. Now if $Y=\operatorname{Spec}\left(H_{\mathbb{C}}\left(T_{d}(k), T_{d}(\mathcal{O})\right)\right.$ is the complex torus dual to $T_{d}$, then given an irreducible $\pi$ with a $K$-fixed vector the Satake isomorphism produces a $W_{d^{-}}$ orbit of elements of $Y$. Twisting $\pi$ by $\chi$ amounts to changing this orbit by adding to it the $W_{d}$-stable element $y_{\chi}$ of $Y$ corresponding to the character of $T_{d}(k) / T_{d}(\mathcal{O})$ induced by $\chi$. Let us define an element $t_{\chi}$ of $\widehat{T}=X^{*}(T) \otimes \mathbb{C}^{\times}$, the complex torus dual to $T$, by $t_{\chi}=\xi \otimes q^{-s}=\xi \otimes|\varpi|^{s}$ (with $q$ the size of the residue field of $k$ and $\varpi$ a uniformiser). The inclusion $T_{d} \rightarrow T$ induces a surjection $\widehat{T} \rightarrow Y$ and some elementary unravelling shows that it sends $t_{\chi}$ to $y_{\chi}$. It follows that if $r_{\pi}: W_{k} \rightarrow{ }^{L} G$ is the representation of $W_{k}$ corresponding to $\pi$, then $r_{\pi \otimes \chi}(w)=\hat{\xi}\left(|w|^{s}\right) r_{\pi}(w)$, with $\hat{\xi}: \mathbb{C}^{\times} \rightarrow \widehat{T} \subseteq \widehat{G}$ the cocharacter of $\widehat{T}$ corresponding to the character $\xi$ of $T$.

We now come back to the $\mathbf{Q}$-structures on $H_{\mathbb{C}}(G(k), K)$. As we have already mentioned, we have a natural $\mathbb{Q}$-structure on $H_{\mathbb{C}}(G(k), K)$ coming from the $\mathbb{Q}$ valued functions $H_{\mathbb{Q}}(G(k), K)$, and we have another one coming from $\mathbb{Q}\left[X_{*}\left(T_{d}\right)\right]^{W_{d}}$ via the Satake isomorphism. This means that, for a smooth irreducible admissible representation $\pi$ of $G(k)$ with a $K$-fixed vector, there are two (typically distinct) notions of what it means to be "defined over $E$ ", for $E$ a subfield of $\mathbb{C}$. Indeed, if $\pi$ is a smooth admissible irreducible representation of $G(k)$ with a $K$-fixed vector, then $\pi^{K}$ is a 1-dimensional complex vector space on which $H_{\mathbb{C}}(G(k), K)$ acts, and this action induces maps

$$
H_{\mathbb{Q}}(G(k), K) \rightarrow \mathbb{C}
$$


and

$$
\mathbb{Q}\left[X_{*}\left(T_{d}\right)\right]^{W_{d}} \rightarrow \mathbb{C} .
$$

Definition 2.2.2. Let $\pi$ be smooth, irreducible and admissible, with a $K$-fixed vector. Let $E$ be a subfield of $\mathbb{C}$.

(i) We say that $\pi$ is defined over $E$ if the induced map $H_{\mathbb{Q}}(G(k), K) \rightarrow \mathbb{C}$ has image lying in $E$.

(ii) We say that the Satake parameter of $\pi$ is defined over $E$ if the induced map $\mathbb{Q}\left[X_{*}\left(T_{d}\right)\right]^{W_{d}} \rightarrow \mathbb{C}$ has image lying in $E$.

If half the sum of the positive roots of $G$ is in the lattice $X^{*}(T)$, then these notions coincide. However there is no reason for them to coincide in general and we shall shortly see examples for $\mathrm{GL}_{2}\left(\mathbb{Q}_{p}\right)$ where they do not.

Note also that it is not immediately clear that these notions are independent of the choice of $K$ : perhaps there is some $\pi$ with a $K$-fixed vector and a $K^{\prime}$-fixed vector for two non-conjugate hyperspecial maximal compacts (for example, the trivial 1dimensional representation of $\mathrm{SL}_{2}\left(\mathbb{Q}_{p}\right)$ has this property), and which is defined over $E$ (or has Satake parameter defined over $E$ ) for one choice but not for the other. The reader should bear in mind that for the time being these notions depend on the choice of $K$, although we will soon see (in Corollary 2.2.4 and Lemma 2.2.5) that they are in fact independent of this choice.

We now discuss some other natural notions of being "defined over $E$ " for $E$ a subfield of $\mathbb{C}$, and relate them to the notions above. So let $E$ be a subfield of $\mathbb{C}$ and let $\pi$ be a smooth irreducible admissible complex representation of $G(k)$ with a $K$-fixed vector, for our fixed choice of $K$. Let $V$ be the underlying vector space for $\pi$.

Lemma 2.2.3. The following are equivalent:

(i) The representation $\pi$ is defined over $E$.

(ii) There is an $E$-subspace $V_{0}$ of $V$ which is $G(k)$-stable and such that $V_{0} \otimes_{E} \mathbb{C}=$ $V$.

(iii) For any (possibly discontinuous) field automorphism $\sigma$ of $\mathbb{C}$ which fixes $E$ pointwise, we have $\pi \cong \pi^{\sigma}=\pi \otimes_{\mathbb{C}, \sigma} \mathbb{C}$ as $\mathbb{C}$-representations.

Proof. That (ii) implies (iii) is clear-it's an abstract representation-theoretic fact. Conversely if (iii) holds, then (ii) follows from Lemma I.1 of WWal85] (note: his $E$ is not our $E$ ), because $V^{K}$ is 1-dimensional. This latter lemma of Waldspurger also shows that if (ii) holds then $V_{0}^{K}$ is 1-dimensional over $E$, and hence (ii) implies (i). To show that (i) implies (ii) we look at the explicit construction giving $\pi$ from the algebra homomorphism $H_{\mathbb{Q}}(G(k), K) \rightarrow \mathbb{C}$ given in [Car79. Given a homomorphism $H_{\mathbb{Q}}(G(k), K) \rightarrow \mathbb{C}$ with image landing in $E$, the resulting spherical function $\Gamma: G(k) \rightarrow \mathbb{C}$ defined in equation (30) of Car79 is also $E$-valued. Now if we define $V_{0}$ to be the $E$-valued functions on $G(k)$ of the form $f(g)=\sum_{i=1}^{n} c_{i} \Gamma\left(g g_{i}\right)$ for $c_{i} \in E$ and $g_{i} \in G(k)$, then $G(k)$ acts on $V_{0}$ by right translations, $V_{0} \otimes_{E} \mathbb{C}$ is the $V_{\Gamma}$ of $\S 4.4$ of Car79, and the arguments in $\S 4.4$ of Car79] show that $\pi \cong V_{0} \otimes_{E} \mathbb{C}$.

Corollary 2.2.4. If $\pi$ is a smooth irreducible admissible unramified representation of $G(k)$, then the notion of being "defined over $E$ " is independent of the choice of hyperspecial maximal compact $K$ for which $\pi^{K}$ is non-zero.

Proof. This is because condition (iii) of Lemma 2.2 .3 is independent of this choice. 
We now prove the analogous result for Satake parameters.

Lemma 2.2.5. If $\pi$ is a smooth irreducible admissible unramified representation of $G(k)$, then the notion of $\pi$ having Satake parameter being defined over $E$ is independent of the choice of hyperspecial maximal compact $K$ for which $\pi^{K} \neq 0$.

Proof. Say $\pi$ is an unramified smooth irreducible admissible representation of $G(k)$ with a $K$-fixed vector. The Satake isomorphism associated to $K$ gives us a character of the algebra $H_{\mathbb{C}}\left(T(k),{ }^{o} T\right)^{W_{d}}$ and hence a $W_{d}$-orbit of complex characters of $T(k)$. Now by p45 of [Bor79] and sections 3 and 4 of [Car79], $\pi$ is a subquotient of the principal series representation attached to any one of these characters, and Theorem 2.9 of [BZ77] then implies that this $W_{d}$-orbit of complex characters are the only characters for which $\pi$ occurs as a subquotient of the corresponding induced representations. Hence the $W_{d}$-orbit of characters, and hence the map $\mathbb{Q}\left[X_{*}\left(T_{d}\right)\right]^{W_{d}} \rightarrow \mathbb{C}$ attached to $\pi$, does not depend on the choice of $K$ in the case when $\pi$ has fixed vectors for more than one conjugacy class of hyperspecial maximal compact. In particular the image of $\mathbb{Q}\left[X_{*}\left(T_{d}\right)\right]^{W_{d}}$ in $\mathbb{C}$ is well-defined independent of the choice of $K$, and hence the notion of having Satake parameter defined over $E$ is also independent of the choice of $K$.

To clarify the meaning of having a Satake parameter defined over $E$, we now explain that in the case of $G=\mathrm{GL}_{n}$ the notion becomes a more familiar one. If $\pi$ is an unramified representation of $\mathrm{GL}_{n}(k)$ and we choose our Borel to be the upper triangular matrices and our torus to be the diagonal matrices, then the formalism above associates to $\pi$ an algebra homomorphism $\mathbb{C}\left[X_{*}\left(T_{d}\right)\right]^{W_{d}} \rightarrow \mathbb{C}$. But here $T=T_{d}$ is the diagonal matrices, and $W_{d}$ is the usual Weyl group $W$ of $G$. The ring $\mathbb{C}\left[X_{*}\left(T_{d}\right)\right]=\mathbb{C}\left[X_{*}(T)\right]$ is then just the ring of functions on the dual torus $\widehat{T}$, and hence an unramified $\pi$ gives rise to a $W_{d}$-orbit on $\widehat{T}$, which can be interpreted as a semisimple conjugacy class $S_{\pi}$ in $\mathrm{GL}_{n}(\mathbb{C})$.

Lemma 2.2.6. Let $G$ be the group $\mathrm{GL}_{n} / k$ and let $\pi$ be an unramified representation of $G(k)$. Let $E$ be a subfield of $\mathbb{C}$. Then the following are equivalent:

(i) The Satake parameter of $\pi$ is defined over $E$.

(ii) The conjugacy class $S_{\pi}$ is defined over $E$.

(iii) The conjugacy class $S_{\pi}$ contains an element of $\mathrm{GL}_{n}(E)$.

Proof. The statement that the Satake parameter is defined over $E$ is precisely the statement that the induced map $\mathbb{Q}\left[X_{*}(T)\right]^{W} \rightarrow \mathbb{C}$ takes values in $E$, which is the statement that the characteristic polynomial of an element of $S_{\pi}$ has coefficients in $E$. Hence (i) and (ii) are equivalent. Furthermore, (ii) is equivalent to (iii) because given a monic polynomial with coefficients in $E$ it is easy to construct a semisimple matrix with this polynomial as its characteristic polynomial.

We leave to the reader the following elementary checks. Let $G_{1}$ and $G_{2}$ be unramified connected reductive groups over $k$, and let $\pi_{1}, \pi_{2}$ be unramified representations of $G_{1}(k), G_{2}(k)$. Then $\pi:=\pi_{1} \otimes \pi_{2}$ is an unramified representation of $\left(G_{1} \times G_{2}\right)(k)$. One can check that $\pi$ is defined over $E$ iff $\pi_{1}$ and $\pi_{2}$ are defined over $E$, and that $\pi$ has Satake parameter defined over $E$ iff $\pi_{1}$ and $\pi_{2}$ do. Now say $k_{1} / k$ is a finite unramified extension of non-archimedean local fields, and $G / k_{1}$ is unramified connected reductive, and set $H=\operatorname{Res}_{k_{1} / k}(G)$. Then $H$ is unramified over $k_{1}$, and if $\pi$ is a representation of $G\left(k_{1}\right)=H(k)$ then $\pi$ is unramified 
as a representation of $G\left(k_{1}\right)$ if and only if it is unramified as a representation of $H(k)$. Furthermore, the two notions of being defined over $E$ (one for $G$ and one for $H$ ) coincide. Moreover, the two notions of having Satake parameter defined over $E$ - one for $G$ and one for $H$-also coincide; we give the argument for this as it is a little trickier. Let $T_{d}$ denote a maximal split torus in $G$ and let $T$ denote its centralizer. The Satake homomorphism for $G$ is an injective ring homomorphism from an unramified Hecke algebra for $G$ into $\mathbb{C}\left[T\left(k_{1}\right) / U\right]$, with $U$ a maximal compact subgroup of $T\left(k_{1}\right)$. The Satake homomorphism for $H$ is a map between the same two rings, and it can be easily checked from the construction in Theorem 4.1 of Car79] that it is in fact the same map. The homomorphism for $G$ is an isomorphism onto the subring $\mathbb{C}\left[T\left(k_{1}\right) / U\right]^{W(G)}$ of $\mathbb{C}\left[T\left(k_{1}\right) / U\right]$, with $W(G)$ the relative Weyl group for the pair $\left(G, T_{d}\right)$. The homomorphism for $H$ is an isomorphism onto $\mathbb{C}\left[T\left(k_{1}\right) / U\right]^{W(H)}$, and hence $\mathbb{C}\left[T\left(k_{1}\right) / U\right]^{W(G)}=\mathbb{C}\left[T\left(k_{1}\right) / U\right]^{W(H)}$. Now intersecting with $\mathbb{Q}\left[T\left(k_{1}\right) / U\right]$ we deduce that $\mathbb{Q}\left[T\left(k_{1}\right) / U\right]^{W(G)}=\mathbb{Q}\left[T\left(k_{1}\right) / U\right]^{W(H)}$ and hence the two $\mathbb{Q}$-structures - one coming from $G$ and one from $H$-coincide.

We finish this section by noting that the notion of being defined over $E$ does not coincide with the notion of having Satake parameter defined over $E$, if $k=\mathbb{Q}_{p}$ and $G=\mathrm{GL}_{2}$. For example, if $\pi$ is the trivial 1-dimensional representation of $\mathrm{GL}_{2}\left(\mathbb{Q}_{p}\right)$ then $\pi$ is defined over $\mathbb{Q}$ but the Satake parameter attached to $\pi$ has eigenvalues $\sqrt{p}$ and $1 / \sqrt{p}$, so the Satake parameter is not defined over $\mathbb{Q}$ (consider traces) but only over $\mathbb{Q}(\sqrt{p})$. Similarly if $\pi$ is the character $|\operatorname{det}|^{1 / 2}$ of $\mathrm{GL}_{2}\left(\mathbb{Q}_{p}\right)$ then $\pi_{p}$ is not defined over $\mathbb{Q}$ but the Satake parameter of $\pi$ has characteristic polynomial $(X-1)(X-p)$ and hence is defined over $\mathbb{Q}$. This issue of the canonical normalisation of the Satake isomorphism "introducing a square root of $p$ " is essentially the reason that one sees two normalisations of local Langlands for $\mathrm{GL}_{n}$ in the literatureone used for local questions and one used for local-global compatibility. We are not attempting to unify these two notions - indeed, one of the motivations of this paper is to draw the distinction between the two notions and explain what each is good for.

2.3. Local Langlands at infinity. We recall the statements and basic properties of the local Langlands correspondence for connected reductive groups over the real or complex field. In fact we work in slightly more generality, for the following reason: the groups that we will apply the definitions and results of this section to are groups defined over completions of number fields at infinite places. So the following subtlety arises: the completion of a number field at a real infinite place is canonically isomorphic to the reals, however the completion of a number field at a complex place is isomorphic to the complex numbers, but not canonically. Hence we actually work with groups defined over either $\mathbb{R}$ or a degree two extension of $\mathbb{R}$ which will be isomorphic to $\mathbb{C}$ but may or may not be canonically isomorphic to $\mathbb{C}$. Note however that all our representations will be on $\mathbb{C}$-vector spaces - there is no ambiguity in our coefficient fields.

Let $k$ be either the real numbers or an algebraic closure of the real numbers. Let $G$ be a connected reductive group over $k$. Fix an algebraic closure $\bar{k}$ of $k$ and let $T \subseteq B$ be a maximal torus and a Borel subgroup of $G_{\bar{k}}$. If $\pi$ is an irreducible admissible complex representation of $G(k)$ then Langlands associates to $\pi$, in a completely canonical way, a $\widehat{G}(\mathbb{C})$-conjugacy class of admissible homomorphisms $r=r_{\pi}$ from the Weil group $W_{k}=W_{\bar{k} / k}$ of $k$ to ${ }^{L} G(\mathbb{C})$. Let us fix a maximal 
torus $\widehat{T}$ in $\widehat{G}_{\mathbb{C}}$ equipped with an identification $X_{*}(\widehat{T})=X^{*}(T)$. The group $W_{k}$ contains a finite index subgroup canonically isomorphic to $\bar{k}^{\times}$; let us assume that $r\left(\bar{k}^{\times}\right) \subseteq \widehat{T}(\mathbb{C}$ ) (which can always be arranged, possibly after conjugating $r$ by an element of $\widehat{G}(\mathbb{C})$ ). If $\sigma$ and $\tau$ denote the two $\mathbb{R}$-isomorphisms $\bar{k} \rightarrow \mathbb{C}$ then one checks easily that for $z \in \bar{k}^{\times}$we have $r(z)=\sigma(z)^{\lambda_{\sigma}} \tau(z)^{\lambda_{\tau}}$ for $\lambda_{\sigma}, \lambda_{\tau} \in X_{*}(\widehat{T}) \otimes \mathbb{C}$ such that $\lambda_{\sigma}-\lambda_{\tau} \in X_{*}(\widehat{T})$. Note that because we may not want to fix a preferred choice of isomorphism $\bar{k}=\mathbb{C}$, we might sometimes "have no preference between $\lambda_{\sigma}$ and $\lambda_{\tau}{ }^{\prime \prime}$; this makes our presentation diverge slightly from other standard references, where typically one isomorphism is preferred.

Because $\widehat{T}(\mathbb{C})$ is usually not its own normaliser in $\widehat{G}(\mathbb{C})$, there is usually more than one way of conjugating $r\left(\bar{k}^{\times}\right)$into $\widehat{T}(\mathbb{C})$, with the consequence that the pair $\left(\lambda_{\sigma}, \lambda_{\tau}\right) \in\left(X_{*}(\widehat{T}) \otimes \mathbb{C}\right)^{2}$ is not a well-defined invariant of $r_{\pi}$; it is only well-defined up to the (diagonal) action of the Weyl group $W=W(G, T)$ on $\left(X_{*}(\widehat{T}) \otimes \mathbb{C}\right)^{2}$. For notational convenience however we will continue to refer to the elements $\lambda_{\sigma}$ and $\lambda_{\tau}$ of $X_{*}(\widehat{T}) \otimes \mathbb{C}$ and will check that none of our important later definitions depend on the choice we have made.

If $k=\mathbb{R}$ then recall from the construction of the $L$-group that there is an action of $\Gamma_{k}$ on $X^{*}(T) \otimes \mathbb{C}$, and we can ask how our pair $\left(\lambda_{\sigma}, \lambda_{\tau}\right)$ behaves under this action. Note first that $\Gamma_{k}$ also acts on $W$, and the $\Gamma_{k}$-action on $X^{*}(T)$ is $W$-semilinear (i.e. for $\gamma \in \Gamma_{k}$ and $w \in W$ and $x \in X^{*}(T) \otimes \mathbb{C}$ we have $\gamma(w x)=\gamma(w) \gamma(x)$ ); this follows from a careful unwinding of the definitions of these actions. If $c$ is the non-trivial element of $\Gamma_{k}$ then one checks that $\left(c\left(\lambda_{\tau}\right), c\left(\lambda_{\sigma}\right)\right)$ is in the same $W$-orbit as $\left(\lambda_{\sigma}, \lambda_{\tau}\right)$.

If $k$ is isomorphic to $\mathbb{C}$ then $\lambda_{\sigma}$ and $\lambda_{\tau}$ are in general unrelated, subject to their difference being in $X^{*}(T)$.

We can put this information together as follows. Let us go back to the general case $k=\mathbb{R}$ or $k \cong \mathbb{C}$. Let $\operatorname{Hom}_{\text {cts }}(\bar{k}, \mathbb{C})=\{\sigma, \tau\}$ denote the continuous field isomorphisms from $\bar{k}$ to $\mathbb{C}$. The group

$$
\left(X^{*}(T) \otimes \mathbb{C}\right)^{\mathrm{Hom}_{\mathrm{cts}}(\bar{k}, \mathbb{C})}
$$

has a diagonal action of the Weyl group $W$. It also has an action of $\operatorname{Gal}(\bar{k} / k)$ defined using both the action of $\operatorname{Gal}(\bar{k} / k)$ on $X^{*}(T)$ and the action on $\operatorname{Hom}_{\text {cts }}(\bar{k}, \mathbb{C})$. Explicitly, if we think of an element of $\left(X^{*}(T) \otimes \mathbb{C}\right)^{\operatorname{Hom}_{\text {cts }}(\bar{k}, \mathbb{C})}$ as a function $F$ : $\operatorname{Hom}_{\text {cts }}(\bar{k}, \mathbb{C}) \rightarrow X^{*}(T) \otimes \mathbb{C}$, then $\gamma \in \operatorname{Gal}(\bar{k} / k)$ sends $F$ to the function sending $\alpha \in \operatorname{Hom}_{\text {cts }}(\bar{k}, \mathbb{C})$ to $\gamma F(\alpha \gamma)$. We deduce that (for both $k=\mathbb{R}$ and $\left.k \cong \mathbb{C}\right)\left(\lambda_{\sigma}, \lambda_{\tau}\right)$ give us a well-defined element of

$$
\left(\left(\left(X^{*}(T) \otimes \mathbb{C}\right)^{\operatorname{Hom}_{\mathrm{cts}}(\bar{k}, \mathbb{C})}\right) / W\right)^{\operatorname{Gal}(\bar{k} / k)},
$$

and hence a well-defined element of

$$
\left(\left(\left(X^{*}(T) \otimes \mathbb{C}\right) / W\right)^{\operatorname{Hom}_{\mathrm{cts}}(\bar{k}, \mathbb{C})}\right)^{\operatorname{Gal}(\bar{k} / k)},
$$

See the comments after Lemma 2.4.1 for a $p$-adic variant of this construction.

The Weyl group orbit of $\left(\lambda_{\sigma}, \lambda_{\tau}\right)$ in $\left(X^{*}(T) \otimes \mathbb{C}\right)^{2}=\left(X^{*}(T) \otimes \mathbb{C}\right)^{\operatorname{Hom}_{\mathrm{cts}}(\bar{k}, \mathbb{C})}$ is naturally an invariant attached to the Weil group representation $r_{\pi}$ rather than to $\pi$ itself, but we can access a large part of it (however, not quite all of it) more intrinsically from $\pi$ using the Harish-Chandra isomorphism. We explain the story 
when $k=\mathbb{R}$; the analogous questions in the case $k \cong \mathbb{C}$ can then be resolved by restriction of scalars.

So, for this paragraph only, we assume $k=\mathbb{R}$. If we regard $G(k)$ as a real Lie group with Lie algebra $\mathfrak{g}$, then our maximal torus $T$ of $G_{\bar{k}}$ gives rise to a Cartan subalgebra $\mathfrak{h}$ of $\mathfrak{g} \otimes_{\mathbb{R}} \bar{k}$. If we now break the symmetry and use $\sigma$ to identify $\bar{k}$ with $\mathbb{C}$, we can interpret the Lie algebra of $T \times_{\bar{k}, \sigma} \mathbb{C}$ as a complex Cartan subalgebra $\mathfrak{h}_{\sigma}^{\mathbb{C}}$ of the complex Lie algebra $\mathfrak{g}^{\mathbb{C}}:=\mathfrak{g} \otimes_{\mathbb{R}} \mathbb{C}$. We have a canonical isomorphism $\mathfrak{h}_{\sigma}^{\mathbb{C}}=X_{*}(T) \otimes_{\mathbb{Z}} \mathbb{C}$ (this isomorphism implicitly also uses $\sigma$, because $X_{*}(T)=\operatorname{Hom}\left(\mathrm{GL}_{1} / \bar{k}, T\right)$ was computed over $\left.\bar{k}\right)$. Now via the HarishChandra isomorphism (normalised in the usual way, so it is independent of the choice of Borel) one can interpret the infinitesimal character of $\pi$ as a $W$-orbit in $\operatorname{Hom}_{\mathbb{C}}\left(\mathfrak{h}_{\sigma}^{\mathbb{C}}, \mathbb{C}\right)=X^{*}(T) \otimes_{\mathbb{Z}} \mathbb{C}=X_{*}(\widehat{T}) \otimes_{\mathbb{Z}} \mathbb{C}$. Furthermore, this $W$-orbit contains $\lambda_{\sigma}$ (this seems to be well-known; see Proposition 7.4 of (Vog93 for a sketch proof). On the other hand, we note that applying this to both $\sigma$ and $\tau$ gives us a pair of $W$-orbits in $X^{*}(T) \otimes \mathbb{C}$, whereas our original construction of $\left(\lambda_{\sigma}, \lambda_{\tau}\right)$ gives us the $W$-orbit of a pair, which is a slightly finer piece of information (which should not be surprising: there are reducible principal series representations of $\mathrm{GL}_{2}(\mathbb{R})$ whose irreducible subquotients (one discrete series, one finite-dimensional) have the same infinitesimal character but rather different associated Weil representations).

We go back now to the general case $k=\mathbb{R}$ or $k \cong \mathbb{C}$. We have a $W$-orbit $\left(\lambda_{\sigma}, \lambda_{\tau}\right)$ in $\left(X^{*}(T) \otimes_{\mathbb{Z}} \mathbb{C}\right)^{\operatorname{Hom}_{\text {cts }}(\bar{k}, \mathbb{C})}$ attached to $\pi$. One obvious "algebraicity" criterion that one could impose on $\pi$ is that $\lambda_{\sigma} \in X_{*}(\widehat{T})=X^{*}(T)$. Note that $\lambda_{\sigma}$ is only welldefined up to an element of the Weyl group, but the Weyl group of course preserves $X_{*}(\widehat{T})=X^{*}(T)$, so the notion is well-defined. Also $\lambda_{\sigma}$ depends on the isomorphism $\sigma: \bar{k} \rightarrow \mathbb{C}$, but if we use $\tau$ instead then the notion remains unchanged, because $\lambda_{\sigma}-\lambda_{\tau} \in X_{*}(\widehat{T})$ and hence $\lambda_{\sigma} \in X_{*}(\widehat{T})$ if and only if $\lambda_{\tau} \in X_{*}(\widehat{T})$. This notion of algebraicity is frequently used in the literature - one can give the connected component of the Weil group of $k$ the structure of the real points of an algebraic group $\mathcal{S}$ over $\mathbb{R}$ and one is asking here that the Weil representation associated to $\pi$ restricts to a map $\mathcal{S}(\mathbb{R}) \rightarrow{ }^{L} G(\mathbb{C})$ induced by a morphism of algebraic groups $\mathcal{S}_{\mathbb{C}} \rightarrow{ }^{L} G_{\mathbb{C}}$ via the inclusion $\mathcal{S}(\mathbb{R}) \subset \mathcal{S}(\mathbb{C})$.

Definition 2.3.1. We say that an admissible Weil group representation $r: W_{k} \rightarrow$ ${ }^{L} G(\mathbb{C})$ is $L$-algebraic if $\lambda_{\sigma} \in X^{*}(T)$. We say that an irreducible representation $\pi$ of $G(k)$ is $L$-algebraic if the Weil group representation associated to it by Langlands is $L$-algebraic.

Note that the notion of $L$-algebraicity for a Weil group representation $r$ depends only on the restriction of $r$ to $\bar{k}^{\times}$, and the notion of $L$-algebraicity for a representation of $G(k)$ depends only on the infinitesimal character of this representation when $k=\mathbb{R}$ (and we shall shortly see that the same is true when $k \cong \mathbb{C}$ ).

Later on we will need the following easy lemma. Say $k=\mathbb{R}$ and $\left(\lambda_{\sigma}, \lambda_{\tau}\right)$ is a representative of the $W$-orbit on $X_{*}(\widehat{T})^{2}$ associated to an $L$-algebraic $\pi_{\infty}$. Regard $\lambda_{\sigma}$ and $\lambda_{\tau}$ as maps $\mathbb{C}^{\times} \rightarrow \widehat{T}$.

Lemma 2.3.2. If $i$ is a square root of -1 in $\bar{k}$ and $j$ is the usual element of order 4 in $W_{k}$ then the element $\alpha_{\infty}:=\lambda_{\sigma}(i) \lambda_{\tau}(i) r_{\pi_{\infty}}(j) \in{ }^{L} G(\mathbb{C})$ has order dividing 2, and its $\widehat{G}(\mathbb{C})$-conjugacy class is well-defined independent of (a) the choice of order of $\sigma$ 
and $\tau,(b)$ the choice of representative $\left(\lambda_{\sigma}, \lambda_{\tau}\right)$ of the $W$-orbit and (c) the choice of square root of -1 in $\bar{k}$.

Proof. Set $r:=r_{\pi}$. We have $\lambda_{\sigma}(z) r(j)=r(j) \lambda_{\tau}(z)$, and $\lambda_{\sigma}(z)$ commutes with $\lambda_{\tau}\left(z^{\prime}\right)$, and from this it is easy to check that $\left(\alpha_{\infty}\right)^{2}=1$ and that $\alpha_{\infty}$ is unchanged if we switch $\sigma$ and $\tau$. Changing representative of the $W$-orbit just amounts to conjugating $r$ by an element of $\widehat{G}(\mathbb{C})$ and hence conjugating $\alpha_{\infty}$ by this same element. Finally one checks easily that conjugating $\alpha_{\infty}$ by $\lambda_{\sigma}(-1)$ gives us the analogous element with $i$ replaced by $-i$.

The notion of $L$-algebraicity will be very important to us later, however it is not hard to find automorphic representations that "appear algebraic in nature" but whose infinite components are not $L$-algebraic. For example one can check that if $E$ is an elliptic curve over $\mathbb{Q}$ and $\pi$ is the associated automorphic representation of $\mathrm{PGL}_{2} / \mathbb{Q}$, then the local component $\pi_{\infty}$, when considered as a representation of $\mathrm{PGL}_{2}(\mathbb{R})$, is not $L$-algebraic: the element $\lambda_{\sigma}$ above is in $X_{*}(\widehat{T}) \otimes_{\mathbb{Z}} \frac{1}{2} \mathbb{Z}$ but not in $X_{*}(\widehat{T})$. What has happened is that the canonical normalisation of the HarishChandra homomorphism involves (at some point in the definition) a twist by half the sum of the positive roots, and it is this twist that has taken us out of the lattice in the elliptic curve example.

This observation motivates a second notion of algebraicity - which it turns out is the one used in Clozel's paper for the group $\mathrm{GL}_{n}$. Let us go back to the case of a general connected reductive $G$ over $k$, either the reals or a field isomorphic to the complexes. Recall that we have fixed $T \subseteq B \subseteq G_{\bar{k}}$ and hence we have the notion of a positive root in $X^{*}(T)$. Let $\delta \in X^{*}(T) \otimes \mathbb{C}$ denote half the sum of the positive roots. We observed above that the assertion " $\lambda_{\sigma} \in X^{*}(T)$ " was independent of the choice of $B$ and of the isomorphism $\bar{k} \cong \mathbb{C}$. But the assertion " $\lambda_{\sigma}-\delta \in X^{*}(T)$ " is also independent of such choices, for if $\lambda_{\sigma}-\delta \in X^{*}(T)$ and $w$ is in the Weyl group, then $w \cdot \lambda_{\sigma}-\delta=w\left(\lambda_{\sigma}-\delta\right)-(\delta-w \cdot \delta) \in X^{*}(T)$, and also $\lambda_{\tau}-\delta=\left(\lambda_{\sigma}-\delta\right)+\left(\lambda_{\tau}-\lambda_{\sigma}\right) \in X^{*}(T)$.

Definition 2.3.3. We say that the admissible Weil group representation $r: W_{k} \rightarrow$ ${ }^{L} G(\mathbb{C})$ is $C$-algebraic if $\lambda_{\sigma}-\delta \in X^{*}(T)$. We say that the irreducible admissible representation $\pi$ of $G(k)$ is $C$-algebraic if the Weil group representation associated to $\pi$ via Langlands' construction is $C$-algebraic.

Again, $C$-algebraicity for $r$ only depends on the restriction of $r$ to $\bar{k}^{\times}$, and $C$ algebraicity for $\pi$ only depends on its infinitesimal character when $k=\mathbb{R}$ (and as we are about to see, the same is true for $k \cong \mathbb{C}$ ).

Here are some elementary remarks about these definitions. If $\delta \in X^{*}(T)$ then the notions of $L$-algebraic and $C$-algebraic coincide. If $G_{1}$ and $G_{2}$ are connected reductive over $k$, if $r_{i}(i=1,2)$ are admissible representations $r_{i}: W_{k} \rightarrow{ }^{L} G_{i}(\mathbb{C})$, then there is an obvious notion of a product $r_{1} \times r_{2}: W_{k} \rightarrow{ }^{L}\left(G_{1} \times G_{2}\right)(\mathbb{C})$ and $r_{1} \times r_{2}$ is $L$-algebraic (resp. $C$-algebraic) iff $r_{1}$ and $r_{2}$ are. One can furthermore check that if $k$ denotes an algebraic closure of the reals and $G / k$ is connected reductive, and if $H=\operatorname{Res}_{k / \mathbb{R}}(G)$, and if $\pi$ is an irreducible admissible representation of $G(k)=H(\mathbb{R})$, then $\pi$ is $L$-algebraic (resp. $C$-algebraic) when considered as a representation of $G(k)$ if and only if it is $L$-algebraic (resp. $C$-algebraic) when considered as a representation of $H(\mathbb{R})$. This assertion comes from a careful reading of sections 4 and 5 of [Bor79]. Indeed, if $T$ is a maximal torus of $G / k$ then $\operatorname{Res}_{k / \mathbb{R}}(T)$ 
is a maximal torus of $H / \mathbb{R}$, and if $\lambda_{\sigma}, \lambda_{\tau} \in X^{*}(T) \otimes \mathbb{C}$ are the parameters attached to a representation of $G(k)$, then $\lambda_{\sigma} \oplus \lambda_{\tau}$ and $\lambda_{\tau} \oplus \lambda_{\sigma} \in\left(X^{*}(T) \oplus X^{*}(T)\right) \otimes \mathbb{C}$ are the parameters attached to the corresponding representation of $H(\mathbb{R})$ (identifying $\widehat{H}(\mathbb{C})$ with $\widehat{G}(\mathbb{C})^{2}$ ), and if $\delta$ is half the sum of the positive roots for $G$ then $\delta \oplus \delta$ is half the sum of the positive roots for $H$. As a consequence, we see that both $L$-algebraicity and $C$-algebraicity of a representation $\pi$ of $G(k)$ are conditions that only depend on the infinitesimal character of the representation of the underlying real reductive group.

Let us again attempt to illustrate the difference between the two notions of algebraicity by considering the trivial 1-dimensional representation of $\mathrm{GL}_{2}(\mathbb{R})$. The Local Langlands correspondence associates to this the 2-dimensional representation $|.|^{1 / 2} \oplus|.|^{-1 / 2}$ of the Weil group of the reals. If we choose the diagonal torus in $\mathrm{GL}_{2}$ and identify its character group with $\mathbb{Z}^{2}$ in the obvious way, then we see that $\lambda_{\sigma}=\lambda_{\tau}=\delta=\left(\frac{1}{2},-\frac{1}{2}\right)$. In particular, $\lambda_{\sigma}$ is not in $X^{*}(T)$, but $\lambda_{\sigma}-\delta$ is, meaning that this representation is $C$-algebraic but not $L$-algebraic. Another example would be the character $|\operatorname{det}|^{1 / 2}$ of $\mathrm{GL}_{2}(\mathbb{R})$; this is associated to the representation $|.| \oplus 1$ of the Weil group, and so $\lambda_{\sigma}=\lambda_{\tau}=(1,0)$ (or $(0,1)$, allowing for the Weyl group action) and on this occasion $\lambda_{\sigma}$ is in $X^{*}(T)$ but $\lambda_{\sigma}-\delta$ is not, hence the representation is $L$-algebraic but not $C$-algebraic. Finally let us consider the discrete series representation of $\mathrm{GL}_{2}(\mathbb{R})$ with trivial central character associated to a weight 2 modular form. The associated representation of the Weil group sends an element $z$ of $\overline{\mathbb{R}}^{\times}$to a matrix with eigenvalues $\sqrt{z \cdot \bar{z}} / z$ and $\sqrt{z \cdot \bar{z}} / \bar{z}$, the square root being the positive square root. We see that the set $\left\{\lambda_{\sigma}, \lambda_{\tau}\right\}$ equals the set $\left\{\left(\frac{1}{2},-\frac{1}{2}\right),\left(-\frac{1}{2}, \frac{1}{2}\right)\right\}$ (with ambiguities due to both the Weyl group action and the two choices of identification of $\overline{\mathbb{R}}$ with $\mathbb{C}$ ) and neither $\lambda_{\sigma}$ nor $\lambda_{\tau}$ are in $X^{*}(T)$, but both of $\lambda_{\sigma}-\delta$ and $\lambda_{\tau}-\delta$ are, so again the representation is $C$-algebraic but not $L$-algebraic.

2.4. The Hodge-Tate cocharacter. In this subsection, let $k$ be a finite extension of the $p$-adic numbers $\mathbb{Q}_{p}$. Let $H$ be a (not necessarily connected) reductive algebraic group over a fixed algebraic closure $\overline{\mathbb{Q}}_{p}$ of $\mathbb{Q}_{p}$. Note that we do not fix an embedding $k \rightarrow \overline{\mathbb{Q}}_{p}$. Let $\bar{k}$ denote an algebraic closure of $k$ and let $\rho: \operatorname{Gal}(\bar{k} / k) \rightarrow H\left(\overline{\mathbb{Q}}_{p}\right)$ denote a continuous group homomorphism. We say that $\rho$ is crystalline/de Rham/Hodge-Tate if for some (and hence any) faithful representation $H \rightarrow \mathrm{GL}_{N}$ over $\overline{\mathbb{Q}}_{p}$, the resulting $N$-dimensional Galois representation is crystalline/de Rham/Hodge-Tate. Let $C$ denote the completion of $\bar{k}$. Then for any continuous injection of fields $i: \overline{\mathbb{Q}}_{p} \rightarrow C$ there is an associated Hodge-Tate cocharacter $\mu_{i}:\left(\mathrm{GL}_{1}\right)_{C} \rightarrow H_{C}$ (where the base extension from $H$ to $H_{C}$ is via $i$ ). We know of no precise reference for the construction of $\mu_{i}$ in this generality; if $H$ were defined over $\mathbb{Q}_{p}$ and $\rho$ took values in $H\left(\mathbb{Q}_{p}\right)$ then $\mu_{i}$ is constructed in [Ser79]. The general case can be reduced to this case in the following way: $H$ descends to group $H_{0}$ defined over a finite extension $E$ of $\mathbb{Q}_{p}$, and a standard Baire category theorem argument shows that $\rho$ takes values in $H_{0}\left(E^{\prime}\right)$ for some finite extension $E^{\prime}$ of $E$. Now let $H_{1}=\operatorname{Res}_{E^{\prime} / \mathbb{Q}_{p}} H_{0}$, so $\rho$ takes values in $H_{1}\left(\mathbb{Q}_{p}\right)$, and Serre's construction of $\mu$ then yields $\mu_{i}$ as above which can be checked to be well-defined independent of the choice of $H_{0}$ and so on via an elementary calculation (do the case $H=\mathrm{GL}_{n}$ first). If $H^{0}$ is the identity component of $H$ and one replaces $\rho$ by $m \rho m^{-1}$, with $m \in H^{0}\left(\overline{\mathbb{Q}}_{p}\right)$, then $\mu_{i}$ becomes $m \mu_{i} m^{-1}$. 
Note that there is a choice of sign that one has to make when defining $\mu_{i}$; we follow Serre so, for example, the cyclotomic character gives rise to the identity map $\mathrm{GL}_{1} \rightarrow \mathrm{GL}_{1}$.

The $H_{C}^{0}$-conjugacy class of $\mu_{i}$ arises as the base extension (via $i$ ) of a cocharacter $\nu_{i}:\left(\mathrm{GL}_{1}\right)_{\mathbb{Q}_{p}} \rightarrow H^{0}$ over $\overline{\mathbb{Q}}_{p}$. Now any $i: \overline{\mathbb{Q}}_{p} \rightarrow C$ is an injection whose image is $\bar{k}$ and hence induces an isomorphism $j={ }^{*} i^{-1 "}: \bar{k} \rightarrow \overline{\mathbb{Q}}_{p}$. We set $\nu_{j}:=\nu_{i}$, an $H^{0}$-conjugacy class of maps $\mathrm{GL}_{1} \rightarrow H$ over $\overline{\mathbb{Q}}_{p}$.

In applications, $H$ will be related to an $L$-group as follows. If $G$ is connected and reductive over $k$, and $\rho: \operatorname{Gal}(\bar{k} / k) \rightarrow{ }^{L} G\left(\overline{\mathbb{Q}}_{p}\right)$ is an admissible representation, then, because $G$ splits over a finite Galois extension $k^{\prime}$ of $k, \rho$ will descend to a representation $\rho: \operatorname{Gal}(\bar{k} / k) \rightarrow \widehat{G}\left(\overline{\mathbb{Q}}_{p}\right) \rtimes \operatorname{Gal}\left(k^{\prime} / k\right)$. The target group can be made into the $\overline{\mathbb{Q}}_{p}$-points of an algebraic group $H$ over $\overline{\mathbb{Q}}_{p}$, and if the associated representation is Hodge-Tate then the preceding arguments associate a $\widehat{G}\left(\overline{\mathbb{Q}}_{p}\right)=$ $H^{0}\left(\overline{\mathbb{Q}}_{p}\right)$-conjugacy class of maps $\nu_{j}: \mathrm{GL}_{1} \rightarrow H_{\overline{\mathbb{Q}}_{p}}$ to each $j: \bar{k} \rightarrow \overline{\mathbb{Q}}_{p}$. If $\widehat{T}$ is a torus in $\widehat{G}$ as usual, then $\nu_{j}$ gives rise to an element of $X_{*}(\widehat{T}) / W=X^{*}(T) / W$, with $T$ a maximal torus of $G_{\bar{k}}$ and $W$ its Weyl group. In particular this construction as $j$ varies gives an element $\nu$ of

$$
\left(X^{*}(T) / W\right)^{\operatorname{Hom}_{\text {cts }}\left(\bar{k}, \overline{\mathbb{Q}}_{p}\right)} .
$$

We are very grateful to Florian Herzig for drawing our attention to this construction and pointing out the formal similarity with the calculations in the previous section.

Just as in the previous section, $\operatorname{Gal}(\bar{k} / k)$ acts on $\left(X^{*}(T) / W\right)^{\operatorname{Hom}_{\mathrm{cts}}\left(\bar{k}, \overline{\mathbb{Q}}_{p}\right)}$ : it acts on each $X^{*}(T) / W$ in the usual way, and it also acts on $\operatorname{Hom}_{\mathrm{cts}}\left(\bar{k}, \overline{\mathbb{Q}}_{p}\right)$ by composition, so if $F: \operatorname{Hom}_{\mathrm{cts}}\left(\bar{k}, \overline{\mathbb{Q}}_{p}\right) \rightarrow X^{*}(T) / W$ and $\gamma \in \mathrm{Gal}(\bar{k} / k)$ we can define $\gamma F$ by $(\gamma F)(\alpha)=\gamma(F(\alpha \gamma))$.

Lemma 2.4.1. $\nu$ is invariant under the action of $\operatorname{Gal}(\bar{k} / k)$.

Proof. If we unravel what is being claimed, we see that it suffices to prove the following. Say $j: \bar{k} \rightarrow \overline{\mathbb{Q}}_{p}$ is a continuous isomorphism, and $\gamma \in \operatorname{Gal}(\bar{k} / k)$. We wish to show that $\nu_{j \gamma}$ is $H^{0}$-conjugate to $\gamma^{-1}\left(\nu_{j}\right)$ which (by the construction of the $L$-group as a semidirect product) is equal to the cocharacter sending $z \in \overline{\mathbb{Q}}_{p}^{\times}$ to $\rho(\gamma)^{-1} \nu_{j}(z) \rho(\gamma)$. The map $j \gamma: \bar{k} \rightarrow \overline{\mathbb{Q}}_{p}$ has an inverse $\gamma^{-1} i: \overline{\mathbb{Q}}_{p} \rightarrow \bar{k}$ which extends to a map $\overline{\mathbb{Q}}_{p} \rightarrow C$. Let $\gamma^{-1} i \rho$ denote the induced map Gal $(\bar{k} / k) \rightarrow H(C)$. If we base extend everything to $C$ via $\gamma^{-1} i$ then we see that our task is to check that $\mu_{\gamma^{-1} i}$ is $H^{0}(C)$-conjugate to the map $z \mapsto\left(\gamma^{-1} i \rho\right)(\gamma)^{-1} \mu_{i}(z)\left(\gamma^{-1} i \rho\right)(\gamma)$. But in fact these two maps are equal. To check this we can reduce to the case $H=$ $\mathrm{GL}_{n} / \overline{\mathbb{Q}}_{p}=\operatorname{Aut}\left(V_{\overline{\mathbb{Q}}_{p}}\right)$, and the result then follows from the commutativity of the following diagram (with $z \in C^{\times}=\mathrm{GL}_{1}(C)$ ).

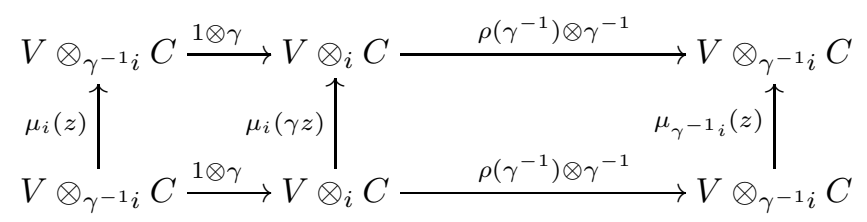


In particular we have

$$
\nu \in\left(\left(X^{*}(T) / W\right)^{\operatorname{Hom}_{\mathrm{cts}}\left(\bar{k}, \overline{\mathbb{Q}}_{p}\right)}\right)^{\operatorname{Gal}(\bar{k} / k)},
$$

analogously to the archimedean case.

\section{GLobal DEFinitions, AND the FirSt CONJECTURES.}

3.1. Algebraicity and arithmeticity. Let $G$ be a connected reductive group defined over a number field $F$. Fix an algebraic closure $\bar{F}$ of $F$ and form the $L$ group ${ }^{L} G=\widehat{G} \rtimes \operatorname{Gal}(\bar{F} / F)$ as in the previous section. For each place $v$ of $F$, fix an algebraic closure $\overline{F_{v}}$ of $F_{v}$, and an embedding $\bar{F} \hookrightarrow \overline{F_{v}}$. Nothing we do depends in any degree of seriousness on these choices - changing them will just change things "by an inner automorphism".

Let $\pi$ be an automorphic representation of $G$. Then we may write $\pi=\otimes_{v}^{\prime} \pi_{v}$, a restricted tensor product, where $v$ runs over all places (finite and infinite) of $F$. Recall that in the previous section we defined notions of $L$-algebraic and $C$-algebraic for certain representations of real and complex groups. We now globalise these definitions.

Definition 3.1.1. We say that $\pi$ is $L$-algebraic if $\pi_{v}$ is $L$-algebraic for all infinite places $v$ of $F$.

Definition 3.1.2. We say that $\pi$ is $C$-algebraic if $\pi_{v}$ is $C$-algebraic for all infinite places $v$ of $F$.

Note that, for $G=\mathrm{GL}_{n}$, the notion of $C$-algebraic coincides (in the isobaric case) with Clozel's notion of algebraic used in Clo90, although for $\mathrm{GL}_{2}$ this choice of normalisation goes back to Hecke. Note also that restriction of scalars preserves both notions: if $K / F$ is a finite extension of number fields and $\pi$ is an automorphic representation of $G / K$ then $\pi$ is $L$-algebraic (resp. $C$-algebraic) when considered as a representation of $G\left(\mathbb{A}_{K}\right)$ if and only if $\pi$ is $L$-algebraic (resp. $C$-algebraic) when considered as a representation of $\operatorname{Res}_{K / F}(G)\left(\mathbb{A}_{F}\right)$. Indeed, this is a local statement and we indicated the proof in $\$ 2.3$.

As examples of these notions, we observe that for Hecke characters of number fields, our notions of $L$-algebraic and $C$-algebraic both coincide with the classical notion of being algebraic or of type $A_{0}$. For $\mathrm{GL}_{2}$ the notions diverge: the trivial 1dimensional representation of $\mathrm{GL}_{2}\left(\mathbb{A}_{\mathbb{Q}}\right)$ is $C$-algebraic but not $L$-algebraic, whereas the representation $|\operatorname{det}|^{1 / 2}$ of $\mathrm{GL}_{2}\left(\mathbb{A}_{\mathbb{Q}}\right)$ is $L$-algebraic but not $C$-algebraic. For $\mathrm{GL}_{3} / \mathbb{Q}$ the notions of $L$-algebraic and $C$-algebraic coincide again (because half the sum of the positive roots in the weight lattice); indeed they coincide for $\mathrm{GL}_{n}$ over a number field if $n$ is odd, and differ by a non-trivial twist if $n$ is even.

The above definitions depend only on the behaviour of $\pi$ at infinite places. The ones below depend only on $\pi$ at the finite places; we remind the reader that the crucial local definitions are given in Definition 2.2.2.

Definition 3.1.3. We say that $\pi$ is $L$-arithmetic if there is a finite subset $S$ of the places of $F$, containing all infinite places and all places where $\pi$ is ramified, and a number field $E \subset \mathbb{C}$, such that for each $v \notin S$, the Satake parameter of $\pi_{v}$ is defined over $E$. 
Definition 3.1.4. We say that $\pi$ is $C$-arithmetic if there is a finite subset $S$ of the places of $F$, containing all infinite places and all places where $\pi$ is ramified, and a number field $E \subset \mathbb{C}$, such that $\pi_{v}$ is defined over $E$ for all $v \notin S$.

Again we note that for $K / F$ a finite extension and $\pi$ an automorphic representation of $G / K, \pi$ is $L$-arithmetic (resp. $C$-arithmetic) if and only if $\pi$ considered as an automorphic representation of $\operatorname{Res}_{K / F}(G)$ is.

Let us consider some examples. An automorphic representation $\pi$ of $\mathrm{GL}_{n} / F$ will be $L$-arithmetic if there is a number field such that all but finitely many of the Satake parameters attached to $\pi$ have characteristic polynomials with coefficients in that number field. So, for example, the trivial 1-dimensional representation of $\mathrm{GL}_{2}\left(\mathbb{A}_{\mathbb{Q}}\right)$ would not be $L$-arithmetic, because the trace of the Satake parameter at a prime $p$ is $p^{1 / 2}+p^{-1 / 2}=\frac{p+1}{\sqrt{p}}$, and any subfield of $\mathbb{C}$ containing $(p+1) / \sqrt{p}$ for infinitely many primes $p$ would also contain $\sqrt{p}$ for infinitely many primes $p$ and hence cannot be a number field. However it would be $C$-arithmetic, because for all primes $p, \pi_{p}$ is the base extension to $\mathbb{C}$ of a representation of $\mathrm{GL}_{2}\left(\mathbb{A}_{\mathbb{Q}}\right)$ on a vector space over $\mathbb{Q}$. Similarly, the representation $|\operatorname{det}|^{1 / 2}$ of $\mathrm{GL}_{2}\left(\mathbb{A}_{\mathbb{Q}}\right)$ is $L$-arithmetic, because all Satake parameters are defined over $\mathbb{Q}$. However this representation is not $C$-arithmetic: each individual $\pi_{p}$ is defined over a number field but there is no number field over which infinitely many of the $\pi_{p}$ are defined, again because such a number field would have to contain the square root of infinitely many primes.

Now let $\pi$ be an arbitrary automorphic representation for an arbitrary connected reductive group $G$ over a number field.

Conjecture 3.1.5. $\pi$ is L-arithmetic if and only if it is L-algebraic.

Conjecture 3.1.6. $\pi$ is $C$-arithmetic if and only if it is $C$-algebraic.

These conjectures are seemingly completely out of reach. The general ideas behind them (although perhaps not the precise definitions we have given) seem to be part of the folklore nowadays, although it is worth pointing out that as far as we know the first person to raise such conjectures explicitly was Clozel in Clo90 in the case $G=\mathrm{GL}_{n}$.

For the group $\mathrm{GL}_{1}$ over a number field both of the conjectures are true; indeed in this case both conjectures say the same thing, the "algebraic implies arithmetic" direction being relatively standard, and the "arithmetic implies algebraic" direction being a non-trivial result in transcendence theory proved by Waldschmidt in Wal81. We prove both conjectures for a general torus in section 4 for the most part by reducing to the case of $\mathrm{GL}_{1}$. On the other hand, neither direction of either conjecture is known for the group $\mathrm{GL}_{2} / \mathbb{Q}$, although in this case the conjectures turn out to be equivalent and there are some partial results in both directions. In particular, Sarnak has shown ( Sar02] that for a Maass form with coefficients in $\mathbb{Z}$, the associated $L$-arithmetic automorphic representation is necessarily $L$-algebraic, and this result was generalised to the case of coefficients in certain quadratic fields in Bru03. Furthermore, if $\pi$ is a cuspidal automorphic representation for $\mathrm{GL}_{2} / \mathbb{Q}$ which is discrete series at infinity, then we show in $\$ 3.3$ that both conjectures hold for $\pi$. However if $\pi$ is principal series at infinity then both directions of both conjectures are in general open.

If one makes conjectures 3.1.5 and 3.1.6 for all groups $G$ simultaneously, then they are in fact equivalent, by the results of section 5 below; for groups with a twisting element (see section 5 for this terminology) this follows from Propositions 
5.2 .2 and 5.2 .3 , and the general case reduces to this one by passage to the covering groups of Section 5 see Proposition 5.3.6.

3.2. Galois representations attached to automorphic representations. We now fix a prime number $p$ and turn to the notion of associating $p$-adic Galois representations to automorphic representations. Because automorphic representations are objects defined over $\mathbb{C}$ and $p$-adic Galois representations are defined over $p$-adic fields, we need a method of passing from one field to the other. We have already fixed an injection $\overline{\mathbb{Q}} \rightarrow \mathbb{C}$; now we fix once and for all a choice of algebraic closure $\overline{\mathbb{Q}}_{p}$ of $\mathbb{Q}_{p}$ and, reluctantly, an isomorphism $\iota: \mathbb{C} \rightarrow \overline{\mathbb{Q}}_{p}$ of "coefficient fields". Recall that our $L$-groups are defined over our fixed algebraic closure $\overline{\mathbb{Q}}$ of $\mathbb{Q}$; our fixed inclusion $\overline{\mathbb{Q}} \rightarrow \mathbb{C}$ then induces, via $\iota$, an embedding $\overline{\mathbb{Q}} \rightarrow \overline{\mathbb{Q}}_{p}$. Ideally we should only be fixing an embedding $\overline{\mathbb{Q}} \rightarrow \overline{\mathbb{Q}}_{p}$, and all our constructions should only depend on the restriction of $\iota$ to $\overline{\mathbb{Q}}$, but of course we cannot prove this. Our choice of $\iota$ does affect matters, in the usual way: for example, if $f=\sum a_{n} q^{n}$ is one of the holomorphic cuspidal newforms for $\mathrm{GL}_{2} / \mathbb{Q}$ of level 1 and weight 24 then 13 splits into two prime ideals in the coefficient field of $f$, and $a_{13}$ is in one of these prime ideals but not the other; hence $f$ will be ordinary with respect to some choices of $\iota$ but not for others. For notational simplicity we drop $\iota$ from our notation but our conjectural association of $p$-adic Galois representations attached to automorphic representations will depend very much on this choice.

We now state two conjectures on the existence of Galois representations attached to $L$-algebraic automorphic representations, the second stronger than the first (in that in specifies a precise set of places at which the Galois representation is unramified/crystalline - this is the only difference between the two conjectures). The first version is the more useful one when formulating conjectures about functoriality. Note that both conjectures depend implicitly on our choice of isomorphism $\iota: \mathbb{C} \rightarrow \overline{\mathbb{Q}}_{p}$ which we use to translate complex parameters to $p$-adic ones.

Conjecture 3.2.1. If $\pi$ is L-algebraic, then there is a finite subset $S$ of the places of $F$, containing all infinite places, all places dividing $p$, and all places where $\pi$ is ramified, and a continuous Galois representation $\rho_{\pi}=\rho_{\pi, \iota}: \operatorname{Gal}(\bar{F} / F) \rightarrow{ }^{L} G\left(\overline{\mathbb{Q}}_{p}\right)$, which satisfies

- The composite of $\rho_{\pi}$ and the natural projection ${ }^{L} G\left(\overline{\mathbb{Q}}_{p}\right) \rightarrow \operatorname{Gal}(\bar{F} / F)$ is the identity map.

- If $v \notin S$, then $\left.\rho_{\pi}\right|_{W_{F_{v}}}$ is $\widehat{G}\left(\overline{\mathbb{Q}}_{p}\right)$-conjugate to $\iota\left(r_{\pi_{v}}\right)$.

- If $v$ is a finite place dividing $p$ then $\left.\rho_{\pi}\right|_{\mathrm{Gal}\left(\overline{F_{v}} / F_{v}\right)}$ is de Rham, and the Hodge-Tate cocharacter of this representation can be explicitly read off from $\pi$ via the recipe in Remark 3.2.3.

- If $v$ is a real place, let $c_{v} \in G_{F}$ denote a complex conjugation at $v$. Then $\rho_{\pi, \iota}\left(c_{v}\right)$ is $\widehat{G}\left(\overline{\mathbb{Q}}_{p}\right)$-conjugate to the element $\iota\left(\alpha_{v}\right)=\iota\left(\lambda_{\sigma_{v}}(i) \lambda_{\tau_{v}}(i) r_{\pi_{v}}(j)\right)$ of Lemma 2.3.2

Conjecture 3.2.2. Assume that $\pi$ is L-algebraic. Let $S$ be the set of the places of $F$ consisting of all infinite places, all places dividing $p$, and all places where $\pi$ is ramified. Then there is a continuous Galois representation $\rho_{\pi, \iota}: \operatorname{Gal}(\bar{F} / F) \rightarrow$ ${ }^{L} G\left(\overline{\mathbb{Q}}_{p}\right)$, which satisfies

- The composite of $\rho_{\pi, \iota}$ and the natural projection ${ }^{L} G\left(\overline{\mathbb{Q}}_{p}\right) \rightarrow \operatorname{Gal}(\bar{F} / F)$ is the identity map. 
- If $v \notin S$, then $\left.\rho_{\pi, l}\right|_{W_{F_{v}}}$ is $\widehat{G}\left(\overline{\mathbb{Q}}_{p}\right)$-conjugate to $\iota\left(r_{\pi_{v}}\right)$.

- If $v$ is a finite place dividing $p$ then $\left.\rho_{\pi, \iota}\right|_{\mathrm{Gal}\left(\overline{F_{v}} / F_{v}\right)}$ is de Rham, and the Hodge-Tate cocharacter associated to this representation is given by the recipe in Remark 3.2.3. Furthermore, if $\pi_{v}$ is unramified then $\left.\rho_{\pi, \iota}\right|_{\mathrm{Gal}\left(\overline{F_{v}} / F_{v}\right)}$ is crystalline.

- If $v$ is a real place, let $c_{v} \in G_{F}$ denote a complex conjugation at $v$. Then $\rho_{\pi, \iota}\left(c_{v}\right)$ is $\widehat{G}\left(\overline{\mathbb{Q}}_{p}\right)$-conjugate to the element $\left.\iota\left(\alpha_{v}\right)=\iota\left(\lambda_{\sigma_{v}}(i) \lambda_{\tau_{v}}(i) r_{\pi_{v}}(j)\right)\right)$ of Lemma 2.3.2.

Remark 3.2.3. The recipe for the Hodge-Tate cocharacter in the conjectures above is as follows. Say $j: \bar{F} \rightarrow \overline{\mathbb{Q}}$ is an isomorphism of fields. We have fixed $\overline{\mathbb{Q}} \rightarrow \mathbb{C}$ and (via $\iota) \overline{\mathbb{Q}} \rightarrow \overline{\mathbb{Q}}_{p}$, so $j$ induces $\bar{F} \rightarrow \overline{\mathbb{Q}}_{p}$ and hence gives us a place $v \mid p$, an algebraic closure $\bar{F}_{v}$ of $F_{v}$ equipped with an identification $j: \bar{F}_{v} \rightarrow \overline{\mathbb{Q}}_{p}$, and a map $\bar{F} \rightarrow \bar{F}_{v}$. Similarly $j$ induces $\bar{F} \rightarrow \mathbb{C}$ and hence gives us an infinite place $w$ of $F$, an algebraic closure $\bar{F}_{w}$ of $F_{w}$ equipped with an identification $\sigma: \bar{F}_{w} \rightarrow \mathbb{C}$, and a map $\bar{F} \rightarrow \bar{F}_{w}$. Now, attached to $\pi_{w}$ and $\sigma$ we have constructed an element $\lambda_{\sigma} \in X^{*}(T) / W$. We need to be careful now (indeed we thank Florian Herzig for pointing out that we were not careful enough in the published version of this paper) - $T$ is a torus of $G$ defined over $\bar{F}_{w}$. Using $\bar{F} \rightarrow \bar{F}_{w}$ we can descend $\lambda_{\sigma}$ to an element of $X^{*}\left(T_{\bar{F}}\right) / W$, and then we can push it out via $\bar{F} \rightarrow \bar{F}_{v}$ to an element of $X^{*}\left(T_{\bar{F}_{v}}\right) / W$. Our conjecture is that this element $\lambda_{\sigma}$ is the Hodge-Tate cocharacter $\nu_{j}$ associated to the embedding $\bar{F}_{v} \rightarrow \overline{\mathbb{Q}}_{p}$.

Remark 3.2.4. The representation $\rho_{\pi, \iota}$ is not necessarily unique up to $\widehat{G}\left(\overline{\mathbb{Q}}_{p}\right)$ conjugation. One rather artificial reason for this is that if $\pi$ is a non-isobaric $L$-algebraic automorphic representation of $\mathrm{GL}_{2} / \mathbb{Q}$ such that $\pi_{v}$ is 1-dimensional for almost all $v$, then there are often many non-semisimple 2-dimensional Galois representations that one can associate to $\pi$ (as well as a semisimple one). But there are other more subtle reasons too. For example if $G$ is a torus over $F$ then the admissible Galois representations into the $L$-group of $G$ are parametrised by $H^{1}(F, \widehat{G})$ (with the Galois group acting on $\widehat{G}$ via the action used to form the $L$-group), and there may be non-zero elements of this group which restrict to zero in $H^{1}\left(F_{v}, \widehat{G}\right)$ for all places $v$ of $F$. If this happens then there is more than one Galois representation that can be associated to the trivial 1-dimensional automorphic representation of $G$. We are grateful to Hendrik Lenstra and Bart de Smit for showing us the following explicit example of a rank 3 torus over $\mathbb{Q}$ where this phenomenon occurs. If $\Gamma$ is the group $(\mathbb{Z} / 2 \mathbb{Z})^{2}$ and $Q$ is the quaternion group of order 8 then $Q$ gives a non-zero element of $H^{2}(\Gamma, \pm 1)$ whose image in $H^{2}\left(\Gamma, \mathbb{C}^{\times}\right)$is non-zero but whose restriction to $H^{2}\left(D, \mathbb{C}^{\times}\right)$is zero for any cyclic subgroup $D$ of $\Gamma$ (consider the corresponding extension of $\Gamma$ by $\mathbb{C}^{\times}$to see these facts). We now "dimension shift". The rank four torus $\mathbb{Z}[\Gamma] \otimes_{\mathbb{Z}} \mathbb{C}^{\times}$has no cohomology in degree greater than zero and has $\mathbb{C}^{\times}$as a subgroup, so the quotient group $T$ is a complex torus with an action of $\Gamma$ and with the property that there's an element of $H^{1}(\Gamma, T)$ whose restriction to any cyclic subgroup is zero. Finally, $\Gamma$ is isomorphic to $\operatorname{Gal}(\mathbb{Q}(\sqrt{13}, \sqrt{17}) / \mathbb{Q}$ ) (a non-cyclic group all of whose decomposition groups are cyclic) and $T$ with its Galois action can be realised as the complex points of the dual group of a torus over $\mathbb{Q}$, giving us our example: there is more than one Galois representation associated to the trivial 1-dimensional representation of this torus. 
Remark 3.2.5. We have normalised local class field theory so that geometric Frobenius elements correspond to uniformisers, and defined our Weil groups accordingly. Had we normalised things the other way (associating arithmetic Frobenius to uniformisers) then the natural thing to do when formulating our unramified local Langlands dictionary would have been to use an arithmetic Frobenius as a generator of our Galois group. In particular our unramified local Langlands dictionary at good finite places would be changed by a non-trivial involution. Had we made this choice initially, conjectures 3.2 .1 and 3.2 .2 need to be modified: one needs to change the Hodge-Tate cocharacter $\mu$ to $-\mu$. However these new conjectures are equivalent to the conjectures as stated, because the required Galois representation predicted by the new conjecture may be obtained directly from $\rho_{\pi}$ by applying the Chevalley involution of ${ }^{L} G$ (the Chevalley involution of $\widehat{G}$ extends to ${ }^{L} G$ and induces the identity map on the Galois group), or indirectly as $\rho_{\tilde{\pi}}$ where $\tilde{\pi}$ is the contragredient of $\pi$. We omit the formal proof that these constructions do the job-in fact, although the arguments at the finite places are not hard, we confess that we were not able to find a precise published reference for the statements at infinity that we need. The point is that we need to know how the local Langlands correspondence for real and complex reductive groups behaves under taking contragredients. The involution on the $\pi$ side induced by contragredient corresponds on the Galois side to the involution on the local Weil representations induced by an involution of the Weil group of the reals/complexes sending $z \in \bar{k}^{\times} \cong \mathbb{C}^{\times}$to $z^{-1}$. It also corresponds to the involution on the Weil representations induced by the Chevalley involution. Both these facts seem to be well-known to the experts but the proof seems not to be in the literature. Jeff Adams and David Vogan inform us that they are working on a manuscript called "The Contragredient" in which these issues will be addressed.

3.3. Example: the groups $\mathrm{GL}_{2} / \mathbb{Q}$ and $\mathrm{PGL}_{2} / \mathbb{Q}$. The following example illustrates the differences between the $C$ - and $L$ - notions in two situations, one where things can be "fixed by twisting" and one where they cannot. The proofs of the assertions made here only involve standard unravelling of definitions and we shall omit them.

Let $\mathbb{A}$ denote the adeles of $\mathbb{Q}$. For $N$ a positive integer, let $K_{0}(N)$ denote the subgroup of $\mathrm{GL}_{2}(\widehat{\mathbb{Z}})$ consisting of matrices which are upper triangular modulo $N$. If $\mathrm{GL}_{2}^{+}(\mathbb{R})$ denotes the matrices in $\mathrm{GL}_{2}(\mathbb{R})$ with positive determinant then $\mathrm{GL}_{2}(\mathbb{A})=$ $\mathrm{GL}_{2}(\mathbb{Q}) K_{0}(N) \mathrm{GL}_{2}^{+}(\mathbb{R})$. Now let $f$ be a modular form of weight $k \geq 2$ which is a normalised cuspidal eigenform for the subgroup $\Gamma_{0}(N)$ of $\mathrm{SL}_{2}(\mathbb{Z})$, and let $s$ denote a complex number. We think of $f$ as a function on the upper half plane. Associated to $f$ and $s$ we define a function $\phi_{s}$ on $\mathrm{GL}_{2}(\mathbb{A})$ by writing an element of $\mathrm{GL}_{2}(\mathbb{A})$ as $\gamma \kappa u$ with $\gamma \in \mathrm{GL}_{2}(\mathbb{Q}), \kappa \in K_{0}(N)$ and $u=\left(\begin{array}{ll}a & b \\ c & d\end{array}\right) \in \mathrm{GL}_{2}^{+}(\mathbb{R})$, and defining $\phi_{s}(\gamma \kappa u)=(\operatorname{det} u)^{k-1+s}(c i+d)^{-k} f((a i+b) /(c i+d))$. This function is well-defined and is a cuspidal automorphic form, which generates an automorphic representation $\pi_{s}$ of $\mathrm{GL}_{2}(\mathbb{A})$. The element $s$ is just a twisting factor; if $s$ is a generic complex number then $\pi_{s}$ will not be algebraic or arithmetic for either of the " $C$ " or " $L$ " possibilities above.

First we consider the arithmetic side of the story. For $p$ a prime not dividing $N$, let $a_{p}$ is the coefficient of $q^{p}$ in the $q$-expansion of $f$. It is well-known that the subfield of $\mathbb{C}$ generated by the $a_{p}$ is a number field $E$. An elementary but long explicit calculation shows the following. If $\pi_{s, p}$ denotes the local component of $\pi_{s}$ at $p$, then $\pi_{s, p}$ has a non-zero invariant vector under the group $\mathrm{GL}_{2}\left(\mathbb{Z}_{p}\right)$ and the action 
of the Hecke operators $T_{p}$ and $S_{p}$ on this 1-dimensional space are via the complex numbers $p^{2-k-s} a_{p}$ and $p^{2-k-2 s}$. The Satake parameter associated to $\pi_{s, p}$ is the semisimple conjugacy class of $\mathrm{GL}_{2}(\mathbb{C})$ consisting of the semisimple elements with characteristic polynomial $X^{2}-a_{p} p^{3 / 2-k-s} X+p^{2-k-2 s}$. Hence $\pi_{s}$ is $L$-arithmetic if $s \in \frac{1}{2}+\mathbb{Z}$. In fact one can go further. By the six exponentials theorem of transcendental number theory one sees easily that a complex number $c$ with the property that $p^{c}$ is algebraic for at least three prime numbers $p$ must be rational. Hence if $\pi_{s}$ is $L$-arithmetic then $s$ is rational and (because a number field is only ramified at finitely many primes and hence cannot contain the $t$ th root of infinitely many prime numbers for any $t>1$ ) one can furthermore deduce that $2 s \in \mathbb{Z}$. Next one observes that $a_{p}$ must be non-zero for infinitely many primes $p \nmid N$ (because one can apply the Cebotarev density theorem to the $\bmod \ell>2$ Galois representation associated to $f$ and to the identity matrix) and deduce (again because a number field cannot contain the square root of infinitely many primes) that $\pi_{s}$ is $L$-arithmetic iff $s \in \frac{1}{2}+\mathbb{Z}$. Now by Proposition 5.2 .3 (whose proof uses nothing that we haven't already established) we see that $\pi_{s}$ is $C$-arithmetic iff $s \in \mathbb{Z}$.

We now consider the algebraic side of things. If $\pi_{s, \infty}$ denotes the local component of $\pi_{s}$ at infinity and we choose the Cartan subalgebra $\mathfrak{h}^{\mathbb{C}}$ of $\mathfrak{g l}_{2}(\mathbb{C})$ spanned by $H:=\left(\begin{array}{cc}1 & 0 \\ 0 & -1\end{array}\right)$ and $Z:=\left(\begin{array}{ll}1 & 0 \\ 0 & 1\end{array}\right)$ then the infinitesimal character of $\pi_{s, \infty}$ (thought of as a Weil group orbit in $\left.\operatorname{Hom}_{\mathbb{C}}\left(\mathfrak{h}^{\mathbb{C}}, \mathbb{C}\right)\right)$ sends $H$ to $\pm(k-1)$ and $Z$ to $2 s+k-2$. The characters of the torus in $\mathrm{GL}_{2}(\mathbb{R})$ give rise to the lattice $X^{*}(T)$ in $\operatorname{Hom}\left(\mathfrak{h}^{\mathbb{C}}, \mathbb{C}\right)$ consisting of linear maps that send $H$ and $Z$ to integers of the same parity. Hence $\pi_{s}$ is $C$-algebraic iff $s \in \mathbb{Z}$ and $L$-algebraic iff $s \in \frac{1}{2}+\mathbb{Z}$. In particular $\pi_{s}$ is $L$-algebraic iff it is $L$-arithmetic, and $\pi_{s}$ is $C$-algebraic iff it is $C$-arithmetic.

We now play the same game for Maass forms. If $f$ (a real analytic function on the upper half plane) is a cuspidal Maass form of level $\Gamma_{0}(N)$ which is an eigenform for the Hecke operators, and $s \in \mathbb{C}$ then one can define a function $\phi_{s}$ on $G_{2}(\mathbb{A})$ by writing an element of $\mathrm{GL}_{2}(\mathbb{A})$ as $\gamma \kappa u$ as above, writing $u=\left(\begin{array}{ll}a & b \\ c & d\end{array}\right)$, and defining $\phi_{s}(\gamma \kappa u)=\operatorname{det}(u)^{s} f((a i+b) /(c i+d))$. If we now assume that $f$ is the Maass form associated by Langlands and Tunnell to a Galois representation $\rho: \operatorname{Gal}(\overline{\mathbb{Q}} / \mathbb{Q}) \rightarrow$ $\mathrm{SL}_{2}(\mathbb{C})$ with solvable image, and if $p \nmid N$ is prime and $a_{p}=\operatorname{tr}\left(\rho\left(\right.\right.$ Frob $\left.\left._{p}\right)\right)$, then the $a_{p}$ generate a number field $E$ (an abelian extension of $\mathbb{Q}$ in this case) and a similar explicit calculation, which again we omit, shows that $\pi_{s}$ is $L$-arithmetic iff $\pi_{s}$ is $L$-algebraic iff $s \in \mathbb{Z}$, and that $\pi_{s}$ is $C$-arithmetic iff $\pi_{s}$ is $C$-algebraic iff $s \in \frac{1}{2}+\mathbb{Z}$.

Note in particular that the answer in the Maass form case is different to the holomorphic case in the sense that $s \in \mathbb{Z}$ corresponded to the $C$-side in the holomorphic case and the $L$-side in the Maass form case.

An automorphic representation for $\mathrm{PGL}_{2} / \mathbb{Q}$ is just an automorphic representation for $\mathrm{GL}_{2} / \mathbb{Q}$ with trivial central character. One checks that the $\pi_{s}$ corresponding to the holomorphic modular form has trivial central character iff $s=1-\frac{k}{2}$ (this is because the form was assumed to have trivial Dirichlet character) and, again because the form has trivial character, $k$ must be even so in particular the $\pi_{s}$ which descends to $\mathrm{PGL}_{2} / \mathbb{Q}$ is $C$-algebraic and $C$-arithmetic. However, the $\pi_{s}$ corresponding to the Maass form with trivial character has trivial central character iff $s=0$, which is $L$-algebraic and $L$-arithmetic. Hence, when applied to the group $\mathrm{PGL}_{2} / \mathbb{Q}$, our conjecture above says that there should be a Galois representation to $\mathrm{SL}_{2}\left(\overline{\mathbb{Q}}_{\ell}\right)$ associated to the Maass form but it says nothing about the holomorphic form. However, the holomorphic form is clearly algebraic in some sense and indeed 
there is a Galois representation associated to the holomorphic form-namely the Tate module of the elliptic curve. Note however that the determinant of the Tate module of an elliptic curve is the cyclotomic character, which is not the square of any 1-dimensional Galois representation (complex conjugation would have to map to an element of order 4) and hence no twist of the Tate module of an elliptic curve can take values in $\mathrm{SL}_{2}\left(\overline{\mathbb{Q}}_{\ell}\right)$. This explains why we have thus far restricted to $L$-algebraic representations for our conjecture attaching Galois representations to automorphic representations.

Finally, we note that it is easy to check that the automorphic representations corresponding to Hilbert modular forms with weights that are not all congruent modulo 2, are neither $C$-algebraic nor $L$-algebraic (cf. pp.91-92 of [Clo90]).

3.4. Why $C$-algebraic? Our conjecture above only attempts to associate Galois representations to $L$-algebraic automorphic representations. So why consider $C$ algebraic representations at all? For $\mathrm{GL}_{n}$ the issue is only one of twisting: $\pi$ is $L$-algebraic iff $\pi$.| $\left.\operatorname{det}()\right|^{.(n-1) / 2}$ is $C$-algebraic. Furthermore, for groups such as $\mathrm{SL}_{2}$ in which half the sum of the positive roots is in $X^{*}(T)$, the notions of $L$-algebraic and $C$-algebraic coincide. On the other hand, as the previous example of $\mathrm{PGL}_{2} / \mathbb{Q}$ attempted to illustrate, one does not always have this luxury of being able to pass easily between $L$-algebraic and $C$-algebraic representations for a given group $G$. Furthermore a lot of naturally occurring representations are $C$-algebraic: for example any cohomological automorphic representation will always be $C$-algebraic (see Lemma 7.2 .2 below) and, as the case of $\mathrm{PGL}_{2} / \mathbb{Q}$ illustrated, there may be natural candidates for Galois representations associated to these automorphic representations, but they may not take values in the $L$-group of $G$ ! In fact, essentially all known examples of Galois representations attached to automorphic representations ultimately come from the cohomology of Shimura varieties (although in some cases the constructions also use congruence arguments), and this cohomology is naturally decomposed in terms of cohomological automorphic representations. Much of the rest of this paper is devoted to examining the relationship between $C$-algebraic and $L$-algebraic in greater detail, and defining a " $C$-group", which should conjecturally receive the Galois representations attached to $C$-algebraic automorphic representations.

\section{THE CASE OF TORI.}

4.1. In this section we prove conjectures 3.1.5, 3.1.6, 3.2.1 and 3.2.2 when $G$ is a torus over a number field $F$. That we can do this should not be considered surprising. Indeed, if $G=\mathrm{GL}_{1}$ then the results have been known for almost 30 years, and the general case can be reduced to the $\mathrm{GL}_{1}$ case via base change and a $p$-adic version of results of Langlands on the local and global Langlands correspondence for tori. Unfortunately we have not been able to find a reference which does what we want so we include some of the details here.

First note that if $G$ is a torus then half the sum of the positive roots is zero, so the Satake isomorphism preserves $\mathbb{Q}$-structures and hence the notions of $C$-arithmetic and $L$-arithmetic coincide and we can use the phrase "arithmetic" to denote either of these notions. Furthermore, again because half the sum of the positive roots is zero, the notions of $C$-algebraic and $L$-algebraic also coincide, and we can use the phrase "algebraic" to mean either of these two notions (and in the case $G=\mathrm{GL}_{1} / F$ 
this coincides with the classical definition, and with Weil's notion of being of type $\left.\left(A_{0}\right)\right)$.

Recall that to give a torus $G / F$ is to give its character group, which (after choosing an $\bar{F}$ ) is a finite free $\mathbb{Z}$-module equipped with a continuous action of $\operatorname{Gal}(\bar{F} / F)$. Let $K \subset \bar{F}$ denote a finite Galois extension of $F$ which splits $G$; then this action of $\operatorname{Gal}(\bar{F} / F)$ factors through $\operatorname{Gal}(K / F)$. An automorphic representation of $G / F$ is just a continuous group homomorphism $G(F) \backslash G\left(\mathbb{A}_{F}\right) \rightarrow \mathbb{C}^{\times}$.

Let $B C$ denote the usual base change map from automorphic representations of $G / F$ to automorphic representations of $G / K$, induced by the norm map $N$ : $G\left(\mathbb{A}_{K}\right) \rightarrow G\left(\mathbb{A}_{F}\right)$.

Lemma 4.1.1. If $\pi$ is an automorphic representation of $G / F$ then $\pi$ is algebraic if and only if $B C(\pi)$ is.

Proof. This is a local statement, and if we translate it over to a statement about representations of Weil groups then it says that if $k$ is an algebraic closure of $\mathbb{R}$ then $r: W_{\mathbb{R}} \rightarrow{ }^{L} G(\mathbb{C})$ is algebraic iff its restriction to $W_{k}$ is, which is clear because our definition of algebraicity of $r$ only depended on the restriction of $r$ to $W_{k}$.

Now let $T$ denote a torus over a local field $k$, and assume $T$ splits over an unramified extension of $k$. The topological group $T(k)$ has a unique maximal compact subgroup $U$. Let $\chi$ be a continuous group homomorphism $T(k) \rightarrow \mathbb{C}^{\times}$with $U$ in its kernel. Note that $U$ is hyperspecial and hence $\chi$ is unramified.

Lemma 4.1.2. For $E$ a subfield of $\mathbb{C}$, the following are equivalent:

(i) $\chi$ is defined over $E$.

(ii) The Satake parameter of $\chi$ is defined over $E$.

(iii) The image of $\chi$ is contained in $E^{\times}$.

Proof. The Satake isomorphism in this situation is simply the identity isomorphism $\mathbb{C}[T(k) / U]=\mathbb{C}[T(k) / U]$, which induces the identity isomorphism $\mathbb{Q}[T(k) / U]=$ $\mathbb{Q}[T(k) / U]$, so (i) and (ii) are equivalent. The equivalence of (i) and (iii) follows from the statement that $\chi: T(k) / U \rightarrow \mathbb{C}^{\times}$is $E^{\times}$-valued if and only if the induced ring homomorphism $\mathbb{Q}[T(k) / U] \rightarrow \mathbb{C}$ is $E$-valued.

If $k_{1} / k$ is a finite extension of local fields and if $T / k$ is a torus then we also use the notation $\mathrm{BC}$ to denote the map $\operatorname{Hom}\left(T(k), \mathbb{C}^{\times}\right) \rightarrow \operatorname{Hom}\left(T\left(k_{1}\right), \mathbb{C}^{\times}\right)$induced by the norm map $N: T\left(k_{1}\right) \rightarrow T(k)$. Now suppose again that $T$ is an unramified torus over $k$ and $\chi: T(k) \rightarrow \mathbb{C}^{\times}$is an unramified character.

Corollary 4.1.3. If $\chi$ is defined over $E$ and if $k_{1} / k$ is a finite extension, then $\mathrm{BC}(\chi)$ is defined over $E$.

Proof. The image of $\mathrm{BC}(\chi)$ is contained in the image of $\chi$.

We now go back to the global situation. Let $\pi$ denote an automorphic representation of $G / F$, with $G$ a torus, and let $\mathrm{BC}(\pi)$ denote its base change to $G / K$, where $K$ is a finite Galois extension of $F$ which splits $G$.

Corollary 4.1.4. If $\pi$ is arithmetic then $\mathrm{BC}(\pi)$ is arithmetic.

Proof. Immediate from the previous corollary.

Theorem 4.1.5. If $G$ is a split torus over a number field, then the notions of arithmetic and algebraic coincide. 
Proof. The fact that algebraic implies arithmetic is standard; the other implication is Théorème 5.1 of [Wal82] (which uses a non-trivial result in transcendence theory).

Corollary 4.1.6. If $G$ is a torus over a number field $F$ and $\pi$ is an automorphic representation of $G$, and $\pi$ is arithmetic, then $\pi$ is algebraic.

Proof. If $\pi$ is arithmetic then its base change to $K$ (a splitting field for $G$ ) is arithmetic (by Corollary 4.1.4), and hence algebraic by the previous theorem. Hence $\pi$ is algebraic by Lemma 4.1.1,

To show that algebraic automorphic representations for $G$ are arithmetic, we give a re-interpretation of what it means for an automorphic representation of a torus to be algebraic; we are grateful to Ambrus Pál for pointing out to us that such a re-interpretation should exist. First some notation. Let $F_{\infty}:=F \otimes_{\mathbb{Q}} \mathbb{R}$. Let $\Sigma$ denote the set of embeddings $\sigma: F \hookrightarrow \overline{\mathbb{Q}}$. Recall that because we have fixed an embedding $\overline{\mathbb{Q}} \rightarrow \mathbb{C}$, each $\sigma \in \Sigma$ can be regarded as an embedding $F \rightarrow \mathbb{C}$, and hence induces maps $F_{\infty} \rightarrow \mathbb{C}$ and $\sigma_{\infty}: G\left(F_{\infty}\right) \rightarrow G_{\sigma}(\mathbb{C})$, where $G_{\sigma}$ is the group over $\overline{\mathbb{Q}}$ induced from $G$ via base extension via $\sigma$.

Proposition 4.1.7. The representation $\pi$ is algebraic if and only if for each $\sigma \in \Sigma$ there is an algebraic character $\lambda_{\sigma}: G_{\sigma} \rightarrow \mathrm{GL}_{1} / \overline{\mathbb{Q}}$ such that $\pi$ agrees with $\prod_{\sigma \in \Sigma} \lambda_{\sigma} \circ$ $\sigma_{\infty}$ on $G\left(F_{\infty}\right)^{0}$ (the identity component of the Lie group $G\left(F_{\infty}\right)$ ).

Proof. This statement is local at infinity, and can be checked by "brute force", explicitly working out what the local Langlands correspondence for tori over the reals and complexes is and noting that it is true in every case.

Corollary 4.1.8. If $\pi$ is an algebraic automorphic representation of $G / F$, and if we write $\pi=\pi_{f} \times \pi_{\infty}$, with $\pi_{\infty}: G\left(F_{\infty}\right) \rightarrow \mathbb{C}^{\times}$, then $\pi_{\infty}(G(F))$ is contained within a number field.

Proof. By the preceding proposition we know that $\left.\pi_{\infty}\right|_{G(F)}$ is the product of a character of order at most 2 by a continuous group homomorphism $G(F) \rightarrow \mathbb{C}^{\times}$ which is the product of maps $\phi_{\sigma}: G(F) \stackrel{\sigma}{\rightarrow} G_{\sigma}(\mathbb{C}) \rightarrow \mathbb{C}^{*}$ given by composing an algebraic character with an embedding $\sigma: F \hookrightarrow \mathbb{C}$. Hence it suffices to prove that $\phi(G(F))$ is contained within a number field for such a $\phi$. However both $T$ and $\mathbb{G}_{m}$ are defined over $F$, so the character descends to some number field $L$, which we may assume splits $T$ and contains the images of all embeddings $F \hookrightarrow \mathbb{C}$. But then $\phi(F) \subset \phi(L) \subset L^{\times}$, as required.

Theorem 4.1.9. The notions of arithmetic and algebraic coincide for automorphic representations of tori over number fields.

Proof. Let $G / F$ be a torus over a number field, and let $\pi$ be an automorphic representation of $G$. By Corollary 4.1.6 we know that if $\pi$ is arithmetic then $\pi$ is algebraic, so we only have to prove the converse. We will make repeated use of the trivial observation (already used above) that if $X$ is a finite index subgroup of an abelian group $Y$, then the image of a character of $Y$ is contained in a number field if and only if the image of its restriction to $X$ is contained in a (possibly smaller) number field.

Let $\pi=\otimes_{v} \pi_{v}$ be algebraic. If $K$ is a finite Galois extension of $F$ splitting $G$ then $B C_{K / F}(\pi)$ is algebraic by Lemma 4.1 .1 and hence arithmetic by Theorem 
4.1.5. Hence there is a number field $E$ and some finite set $S_{K}$ of places of $K$, containing all the infinite places, such that for $w \notin S_{K}, \mathrm{BC}(\pi)_{w}$ is defined over $E$, and hence $\mathrm{BC}(\pi)_{w}$ has image in $E^{\times}$. By increasing $S_{K}$ if necessary, we can assume that $S_{K}$ is precisely the set of places of $K$ lying above a finite set $S$ of places of $F$.

Let $N: G\left(\mathbb{A}_{K}\right) \rightarrow G\left(\mathbb{A}_{F}\right)$ denote the norm map. Standard results from global class field theory (see for example p.244 of [Lan97] for the crucial argument) imply that $G(F) N\left(G\left(\mathbb{A}_{K}\right)\right)$ is a closed and open subgroup of finite index in $G\left(\mathbb{A}_{F}\right)$. Hence if $\mathbb{A}_{F}^{S}$ denotes the restricted product of the completions of $F$ at places not in $S$, and $\mathbb{A}_{K}^{S_{K}}$ denotes the analogous product for $K$, then $G(F) N\left(G\left(\mathbb{A}_{K}^{S_{K}}\right)\right)$ has finite index in $G\left(\mathbb{A}_{F}^{S}\right)$. Let $\pi^{S}: G\left(\mathbb{A}_{F}^{S}\right) \rightarrow \mathbb{C}^{\times}$denote the restriction of $\pi$ to $G\left(\mathbb{A}_{F}^{S}\right)$. Then $\pi=\pi^{S} \cdot \prod_{v \in S, v \nmid \infty} \pi_{v} . \pi_{\infty}$. We know that $\pi$ is trivial on $G(F)$ (by definition) and that $\pi_{\infty}$ sends $G(F)$ to a number field (by Corollary 4.1.8). We also know that $\pi_{v}\left(G\left(F_{v}\right)\right.$ ) (and thus $\pi_{v}(G(F))$ is contained within a number field for each finite place $v \in S$ (because $B C_{K / F}(\pi)$ is arithmetic, we know that $\pi_{v}\left(N\left(K_{w}\right)\right.$ ) is contained in a number field, where $w \mid v$ is a place of $K$, and $N\left(K_{w}\right)$ has finite index in $\left.F_{v}\right)$. Hence $\pi^{S}(G(F))$ is contained within a number field. Then since $G(F) N\left(G\left(\mathbb{A}_{K}^{S_{K}}\right)\right)$ has finite index in $G\left(\mathbb{A}_{F}^{S}\right)$, we deduce that $\pi\left(G\left(\mathbb{A}_{F}^{S}\right)\right)$ is contained within a number field, and hence $\pi$ is arithmetic, as required.

What remains now is to prove Conjecture 3.2 .2 (which of course implies Conjecture 3.2.1). This follows straightforwardly from Langlands' proof of the Langlands correspondence for tori, and the usual method for associating Galois representations to algebraic representations of $\mathbb{G}_{m}$. Take $\pi, G, F$ and $K$ as above, and again let $\Sigma$ denote the field embeddings $F \rightarrow \overline{\mathbb{Q}}$, noting now that because of our fixed embedding $\overline{\mathbb{Q}} \subset \overline{\mathbb{Q}}_{p}$ we can also interpret each element of $\Sigma$ as a field embedding $F \rightarrow \overline{\mathbb{Q}}_{p}$.

The first step is to associate a " $p$-adic automorphic representation"- a continuous group homomorphism $\pi_{p}: G(F) \backslash G\left(\mathbb{A}_{F}\right) \rightarrow \overline{\mathbb{Q}}_{p}^{\times}$-to $\pi$, which we do by mimicking the standard construction in the split case. For $\sigma \in \Sigma$ recall that $\sigma_{\infty}$ is the induced map $G\left(F_{\infty}\right) \rightarrow G_{\sigma}(\mathbb{C})$. By Proposition 4.1.7 there are characters $\lambda_{\sigma} \in X^{*}\left(G_{\sigma}\right)$ (regarded here as maps $G_{\sigma}(\mathbb{C}) \rightarrow \mathbb{C}^{\times}$) for each $\sigma \in \Sigma$ with the property that

$$
\left.\pi\right|_{G\left(F_{\infty}\right)^{0}}=\prod_{\sigma \in \Sigma} \lambda_{\sigma} \circ \sigma_{\infty} .
$$

The right hand side of the above equation can be regarded as a character of $G\left(F_{\infty}\right)$ and hence as a character $\lambda_{\infty}$ of $G\left(\mathbb{A}_{F}\right)$, trivial at the finite places. Define $\pi^{\text {alg }}=$ $\pi / \lambda_{\infty}$, a continuous group homomorphism $G\left(\mathbb{A}_{F}\right) \rightarrow \mathbb{C}^{\times}$trivial on $G\left(F_{\infty}\right)^{0}$ but typically non-trivial on $G(F)$. However $\pi^{\text {alg }}(G(F))$ is contained within a number field by Corollary 4.1.8 and it is now easy to check that the image of $\pi^{\text {alg }}$ is contained within $\overline{\mathbb{Q}}$. We now regard $\pi^{\text {alg }}$ as taking values in $\overline{\mathbb{Q}}_{p}$ via our fixed embedding $\overline{\mathbb{Q}} \subset \overline{\mathbb{Q}}_{p}$.

Now, let $F_{p}=F \otimes_{\mathbb{Q}} \mathbb{Q}_{p}$, and note that every $\sigma: F \rightarrow \overline{\mathbb{Q}}_{p}$ in $\Sigma$ induces a map $F_{p} \rightarrow \overline{\mathbb{Q}}_{p}$ and hence a map $\sigma_{p}: G\left(F_{p}\right) \rightarrow G_{\sigma}\left(\overline{\mathbb{Q}}_{p}\right)$. Each $\lambda_{\sigma}$ can be regarded as a $\operatorname{map} G_{\sigma}\left(\overline{\mathbb{Q}}_{p}\right) \rightarrow \overline{\mathbb{Q}}_{p}^{\times}$, and hence the product

$$
\lambda_{p}:=\prod_{\sigma} \lambda_{\sigma} \circ \sigma_{p}
$$


is a continuous group homomorphism $G\left(F_{p}\right) \rightarrow \overline{\mathbb{Q}}_{p}^{\times}$and can also be regarded as a continuous group homomorphism $G\left(\mathbb{A}_{F}\right) \rightarrow \overline{\mathbb{Q}}_{p}^{\times}$, trivial at all places other than those above $p$. The crucial point, which is easy to check, is that the product $\pi_{p}:=\pi^{\text {alg }} \lambda_{p}$ is a continuous group homomorphism $G\left(\mathbb{A}_{F}\right) \rightarrow \overline{\mathbb{Q}}_{p}^{\times}$which is trivial on $G(F)$.

Now, in Theorem 2(b) of [Lan97], Langlands proves that there is a natural surjection with finite kernel from the set of $\widehat{G}(\mathbb{C})$-conjugacy classes of continuous homomorphisms from the Weil group $W_{F}$ to ${ }^{L} G(\mathbb{C})$ to the set of continuous homomorphisms from $G(F) \backslash G\left(\mathbb{A}_{F}\right)$ to $\mathbb{C}^{\times}$(that is, the set of automorphic representations of $G / F)$, compatible with the local Langlands correspondence at every place. His proof starts by establishing a natural surjection from the analogous sets with the continuity conditions removed, and then checking that continuity on one side is equivalent to continuity on the other. However, $\overline{\mathbb{Q}}_{p} \cong \mathbb{C}$ as abstract fields, and one can check that the calculations on pages $243 \mathrm{ff}$ make no use of any particular features of the topology of $\mathbb{C}^{\times}$, and hence apply equally well to continuous homomorphisms $W_{F} \rightarrow{ }^{L} G\left(\overline{\mathbb{Q}}_{p}\right)$ and continuous characters $G(F) \backslash G\left(\mathbb{A}_{F}\right) \rightarrow \overline{\mathbb{Q}}_{p}^{\times}$. Thus $\pi_{p}$ gives a continuous homomorphism (or perhaps several, in which case we simply choose one)

$$
r_{\pi}: W_{F} \rightarrow{ }^{L} G\left(\overline{\mathbb{Q}}_{p}\right),
$$

and by construction we see that for each finite place $v \nmid p$ at which $\pi_{v}=\left(\pi_{p}\right)_{v}$ is unramified, $\left.r_{\pi}\right|_{W_{F_{v}}}$ is $\widehat{G}\left(\overline{\mathbb{Q}}_{p}\right)$-conjugate to $r_{\pi_{v}}$. Again, by construction the composite of $r_{\pi}$ and the natural projection ${ }^{L} G\left(\overline{\mathbb{Q}}_{p}\right) \rightarrow \operatorname{Gal}(\bar{F} / F)$ is just the natural surjection $W_{F} \rightarrow \operatorname{Gal}(\bar{F} / F)$.

Lemma 4.1.10. The representation $r_{\pi}$ of $W_{F}$ factors through the natural surjection $W_{F} \rightarrow \operatorname{Gal}(\bar{F} / F)$.

Proof. The kernel of the natural surjection $W_{F} \rightarrow \operatorname{Gal}(\bar{F} / F)$ is the connected component of the identity in $W_{F}$; but $r_{\pi}$ must vanish on this, because ${ }^{L} G\left(\overline{\mathbb{Q}}_{p}\right)$ is totally disconnected.

We let $\rho_{\pi}$ denote the representation of $\operatorname{Gal}(\bar{F} / F)$ determined by $r_{\pi}$.

Lemma 4.1.11. The representation $\rho_{\pi}$ satisfies all the properties required in the statement of Conjecture 3.2.2.

Proof. We need to check the claimed properties at places dividing $p$ and at real places. For the former, we must firstly check that $\rho_{\pi}$ is de Rham with the correct Hodge-Tate weights. However, it is sufficient to check this after restriction to any finite extension of $F$, and in particular we may choose an extension which splits $G$. The evident compatibility of the construction of $\rho_{\pi}$ with base change then easily reduces us to the split case, which is standard. Similarly, the property of being crystalline may be checked over any unramified extension, and if $\pi_{v}$ is unramified then by definition $G$ splits over an unramified extension of $F_{v}$, and we may again reduce to the split case.

Suppose now that $v$ is a real place of $F$. Recall that the natural surjection $W_{F_{v}} \rightarrow \operatorname{Gal}\left(\overline{F_{v}} / F_{v}\right)$ sends $j$ to complex conjugation, so we need to determine $\left.r_{\pi}\right|_{W_{F_{v}}}(j)$. Let $\sigma_{v}: F \hookrightarrow \mathbb{C}$ denote the embedding corresponding to $v$ (it is unique because $v$ is a real place) and let $\lambda_{v}$ denote the character $\lambda_{\sigma_{v}}$ of $G_{\sigma_{v}}$. Let $\chi_{v}$ denote the map $G\left(F_{v}\right) \rightarrow \mathbb{C}^{\times}$induced by $\sigma_{v}$ and $\lambda_{v}$. Then $\left(\pi_{p}\right)_{v}=\pi_{v} / \chi_{v}$. Applying local 
Langlands we see that the cohomology class in $H^{1}\left(W_{F_{v}}, \widehat{G}\right)$ associated to $\left(\pi_{p}\right)_{v}$ is the difference of those associated to $\pi_{v}$ and $\chi_{v}$ (because local Langlands is an isomorphism of groups in this abelian setting). Furthermore, one can check (either by the construction of the local Langlands correspondence for real tori in section 9.4 of [Bor79], or an explicit case-by-case check) that the cohomology class attached to $\chi_{v}$ is represented by a cocycle which sends $j \in W_{F_{v}}$ to the element $\lambda_{v}(-1)$ of $\widehat{G}$ (where we now view $\lambda_{v}$ as a cocharacter $\mathbb{C}^{\times} \rightarrow \widehat{G}$ ). Our assertion about $\left.r_{\pi}\right|_{W_{F_{v}}}(j)$ now follows immediately from an explicit calculation on cocycles.

\section{Twisting And Gross' $\eta$.}

\subsection{Algebraicity and arithmeticity under central extensions.}

Definition 5.1.1. We will call a central extension

$$
1 \rightarrow \mathbb{G}_{m} \rightarrow G^{\prime} \rightarrow G \rightarrow 1
$$

of algebraic groups over $F$ a $\mathbb{G}_{m}$-extension of $G$.

Note that one can (after making compatible choices of maximal compact subgroups at infinity, and using the fact that $H^{1}\left(F, \mathbb{G}_{m}\right)=0$ by Hilbert 90 ) identify automorphic representations on $G$ with automorphic representations on $G^{\prime}$ which are trivial on $\mathbb{G}_{m}$. We abuse notation slightly and speak about the $\mathbb{G}_{m}$-extension $G^{\prime} \rightarrow G$ interchangeably with the $\mathbb{G}_{m}$-extension $1 \rightarrow \mathbb{G}_{m} \rightarrow G^{\prime} \rightarrow G \rightarrow 1$.

We now consider how our various notions of arithmeticity and algebraicity behave under pulling back via a $\mathbb{G}_{m}$-extension. So say $G^{\prime} \rightarrow G$ is a $\mathbb{G}_{m}$-extension. The induced map $G^{\prime}\left(\mathbb{A}_{F}\right) \rightarrow G\left(\mathbb{A}_{F}\right)$ is a surjection, and if $\pi$ is an automorphic representation of $G\left(\mathbb{A}_{F}\right)$ then the induced representation $\pi^{\prime}$ of $G^{\prime}\left(\mathbb{A}_{F}\right)$ is also an automorphic representation. Furthermore, we have the following compatibilities between $\pi$ and $\pi^{\prime}$.

Lemma 5.1.2. $\pi$ is L-algebraic (resp. C-algebraic, resp. L-arithmetic, resp. $C$ arithmetic) if and only if $\pi^{\prime}$ is.

Proof. Let us start with the $L$ - and $C$-algebraicity assertions. These assertions follow from purely local assertions at infinity: one needs to check that if $k$ is an archimedean local field (a completion of $F$ in the application), and if $\pi$ is a representation of $G(k)$, with $\pi^{\prime}$ the induced representation of $G^{\prime}(k)$, then $\pi$ is $L$-algebraic (resp. $C$-algebraic) if and only if $\pi^{\prime}$ is. These statements can easily be checked using infinitesimal characters. Indeed a straightforward calculation (using an explicit description of the Harish-Chandra isomorphism) shows that if $T^{\prime}$ is a maximal torus in $G^{\prime}$ over the complexes (where we base change $G^{\prime}$ to the complexes via the map $k \rightarrow \mathbb{C}$ induced from $\sigma: \bar{k} \rightarrow \mathbb{C}$ ), and if the image of $T^{\prime}$ in $G$ is $T$ (a maximal torus of $G$ ), and if $\lambda_{\sigma}$ and $\lambda_{\sigma}^{\prime}$ are the elements of $X^{*}(T) \otimes_{\mathbb{Z}} \mathbb{C}$ and $X^{*}\left(T^{\prime}\right) \otimes_{\mathbb{Z}} \mathbb{C}$ corresponding to $\pi$ and $\pi^{\prime}$ as in $\S 2.3$, then the natural map $X^{*}(T) \otimes_{\mathbb{Z}} \mathbb{C} \rightarrow X^{*}\left(T^{\prime}\right) \otimes_{\mathbb{Z}} \mathbb{C}$ sends $\lambda_{\sigma}$ to $\lambda_{\sigma}^{\prime}$ and both results follow easily.

It remains to prove the arithmeticity statements. Again these statements follow from purely local assertions. Let $k$ denote a non-archimedean local field (a nonarchimedean completion of $F$ at which everything is unramified in the application) and let $\pi$ be an irreducible smooth representation of $G(k)$, with $\pi^{\prime}$ the corresponding representation of $G^{\prime}(k)$. Assume $\pi^{\prime}$ and $\pi$ (and hence $G^{\prime}$ and $G$ ) are unramified. The $C$-arithmeticity assertion of the Proposition follows from the assertion that $\pi$ is 
defined over a subfield $E$ of $\mathbb{C}$ iff $\pi^{\prime}$ is; this is however immediate from Lemma 2.2.3. The $L$-arithmeticity statement follows from the assertion that the Satake parameter of $\pi$ is defined over $E$ iff the Satake parameter of $\pi^{\prime}$ is defined over $E$, which is then what remains to be proved. So let $T^{\prime}$ be the centralizer of a maximal split torus in $G^{\prime}$ over $k$, and let $T$ be its image in $G$. Then $T^{\prime}(k) \rightarrow T(k)$ is a surjection by Hilbert 90. As noted in the proof of Lemma 2.2.5. Theorem 2.9 of [BZ77] shows that the $W_{d}$-orbit of complex characters of $T(k)$ determined by the Satake isomorphism applied to $\pi$ are precisely the characters for which $\pi$ occurs as a subquotient of the corresponding induced representations, and the analogous assertion also holds for $\pi^{\prime}$. It follows that the orbit of characters of $T^{\prime}(k)$ corresponding to $\pi^{\prime}$ is precisely the orbit induced from the characters of $T(k)$ via the surjection $T^{\prime}(k) \rightarrow T(k)$. This implies that the Satake parameter of $\pi^{\prime}$ (thought of as a character of $\mathbb{Q}\left[X_{*}\left(T_{d}^{\prime}\right)\right]^{W_{d}}$ ) is induced from the Satake parameter of $\pi$ via a map between the corresponding unramified Hecke algebras which is in fact the obvious map, and our assertion now follows easily.

5.2. Twisting elements. We now explain the relationship between $L$-algebraic and $C$-algebraic automorphic representations for a connected reductive group $G$ over a number field $F$. In particular, we examine the general question of when $L$ algebraic representations can be twisted to $C$-algebraic representations, following an idea of Gross (see Gro99). We show that in general it is always possible to replace $G$ by a $\mathbb{G}_{m}$-extension for which this twisting is possible, and in this way one can formulate general conjectures about the association of Galois representations to $C$-algebraic (and in particular cohomological by Lemma 7.2.2 below) automorphic representations. As usual, let $X^{*}$ denote the character group in the based root datum for $G$, with its Galois action. Let us stress that we always equip $X^{*}$ with the Galois action coming from the construction used to define the $L$-group (which might well not be the same as the "usual" Galois action on $X^{*}(T)$ induced by the Galois action on $T(\bar{F})$, if $T$ is a maximal torus in $G$ which happens to be defined over $F)$.

Definition 5.2.1. We say that an element $\theta \in X^{*}$ is a twisting element if $\theta$ is $\operatorname{Gal}(\bar{F} / F)$-stable and $\left\langle\theta, \alpha^{\vee}\right\rangle=1 \in \mathbb{Z}$ for all simple coroots $\alpha^{\vee}$.

For some groups $G$ there are no twisting elements; for example, if $G=\mathrm{PGL}_{2}$. On the other hand, if $G$ is semi-simple and simply-connected then half the sum of the positive roots is a twisting element. Another case where twisting elements exist are groups $G$ that are split and have simply-connected derived subgroup, for example $G=\mathrm{GL}_{n}$, although in this case half the sum of the positive roots might not be in $X^{*}$.

If $Q$ is the quotient of $G$ by its derived subgroup, then $X^{*}(Q) \subseteq X^{*}$ and the arguments in section II.1.18 of Jan03 show that $X^{*}(Q)=\left(X^{*}\right)^{W}$, where $W$ is the Weyl group of $G_{\bar{F}}$. Furthermore, $X^{*}(Q)$ is $\operatorname{Gal}(\bar{F} / F)$-stable, and the induced action of $\operatorname{Gal}(\bar{F} / F)$ on $X^{*}(Q)$ is precisely the usual action, induced by the Galois action on $Q(\bar{F})$.

Now let $\delta$ denote half the sum of the positive roots of $G$. If $\delta \in X^{*}$ then $\delta$ is a twisting element; but in general we only have $\delta \in \frac{1}{2} X^{*}$. Let $S^{\prime}$ denote the maximal split torus quotient of $G$, so that

$$
X^{*}\left(S^{\prime}\right)=\left(X^{*}\right)^{W, \operatorname{Gal}(\bar{F} / F)} .
$$


Then if $\theta$ is a twisting element, we see that

$$
\theta-\delta \in \frac{1}{2} X^{*}\left(S^{\prime}\right)
$$

Thus we have a character $|\cdot|^{\theta-\delta}$ of $G(F) \backslash G\left(\mathbb{A}_{F}\right)$, defined as the composite

$$
G\left(\mathbb{A}_{F}\right) \longrightarrow S^{\prime}\left(\mathbb{A}_{F}\right) \stackrel{2(\theta-\delta)}{\longrightarrow} \mathbb{A}_{F}^{\times} \stackrel{|\cdot|}{\longrightarrow} \mathbb{R}_{>0} \stackrel{x \mapsto \sqrt{x}}{\longrightarrow} \mathbb{R}_{>0}
$$

The main motivation behind the notion of twisting elements is the following two propositions.

Proposition 5.2.2. If $\theta$ is a twisting element, then an automorphic representation $\pi$ is $C$-algebraic if and only if $\pi \otimes|\cdot|^{\theta-\delta}$ is L-algebraic.

Proof. This is a consequence of condition 10.3(2) of [Bor79], although formally one has to "reverse-engineer" the construction of (using the notation of $§ 10.2$ of loc. cit.) $\alpha \mapsto \pi_{\alpha}$. We sketch the argument using the notation there. The question is local at each infinite place, so let $k$ denote a completion of $F$ at an infinite place. Let $\widetilde{G}$ be the central extension of $G$ described in $\$ 10.2$ of Bor79. The character $|\cdot|^{\theta-\delta}$ induces a character of $G(k)$ and of $\widetilde{G}(k)$. If $Q$ denotes the maximal torus quotient of $\widetilde{G}$ then this character can be extended to a character of $Q(k)$. The associated element of $H^{1}\left(W_{k}, \widehat{Q}\right)$ is the image of an element $\alpha \in H^{1}\left(W_{k}, Z_{L}\right)$, with $Z_{L}$ the centre of $\widehat{G}$, and one checks easily that the character $\pi_{\alpha}$ of $G(k)$ in $\S 10.2$ of Bor79] coincides with $|\cdot|^{\theta-\delta}$. If $T_{0}$ is a maximal torus in $\widetilde{G}$, and $T$ is its image in $G$, then the restriction of $\alpha$ to $\bar{k}^{\times}$is a $Z_{L}$-valued character which, when considered as a $\widehat{T}_{0}$-valued character of $\bar{k}^{\times}$, has image in $\widehat{T}$ and which (via an easy diagram chase) coincides with the restriction to $\bar{k}^{\times}$of the cohomology class associated via local Langlands to the restriction of $|\cdot|^{\theta-\delta}$ to $T(k)$. Hence $a$ on $\bar{k}^{\times}$is the composite of the norm map down to $\mathbb{R}_{>0}$, the square root map, and the cocharacter of $\widehat{T}$ associated to $2(\theta-\delta)$. Twisting $\pi$ by $|\cdot|^{\theta-\delta}$ corresponds to twisting $r_{\pi}$ by $a$ by $10.3(2)$ of [Bor79] and the result follows easily.

Proposition 5.2.3. If $\theta$ is a twisting element, then $\pi$ is $C$-arithmetic if and only if $\pi \otimes|\cdot|^{\theta-\delta}$ is L-arithmetic.

Proof. Again this is a local issue: by Definitions 3.1.3 and 3.1.4 it suffices to check that if $k$ (a completion of $F$ ) is a non-archimedean local field, if $\chi$ denotes the restriction of $|\cdot|^{\theta-\delta}$ to $G(k)$ and if $\pi$ is an unramified representation of $G(k)$, then $\pi$ is defined over a subfield $E$ of $\mathbb{C}$ iff $\pi \otimes \chi$ has Satake parameter defined over the same subfield $E$. This is relatively easy to check: we sketch the details (using the notation of section 2.2). Let $T$ be a maximal torus of $G / k$, with maximal compact subgroup ${ }^{\circ} T$. Then $\chi$ induces an automorphism $i$ of $H_{\mathbb{C}}\left(T(k),{ }^{o} T\right)$ sending $\left.{ }^{\circ} T t^{o} T\right]$ to $\chi(t)\left[^{\circ} T t^{o} T\right]$, and $i$ commutes with the action of the Weyl group $W_{d}$ and hence induces an automorphism $i$ of the $W_{d}$-invariants of this complex Hecke algebra. If $m_{\pi}$ is the complex character of $H\left(T(k),{ }^{o} T\right)^{W_{d}}$ associated to $\pi$ and $m_{\pi \otimes \chi}$ is the character associated to $\pi \otimes \chi$ then one checks easily that $m_{\pi \otimes \chi}=m_{\pi} \circ i$. The other observation we need is that if $K$ is a hyperspecial maximal compact subgroup of $G(k)$ and if $S$ denotes the Satake isomorphism $S: H_{\mathbb{C}}(G(k), K) \rightarrow H_{\mathbb{C}}\left(T(k),{ }^{o} T\right)^{W_{d}}$ then $i \circ S$ maps $H_{\mathbb{Q}}(G(k), K)$ into $H_{\mathbb{Q}}\left(T(k),{ }^{o} T\right)$ (this follows immediately from formula (19) of section 4.2 of [Car79]), and hence into $H_{\mathbb{Q}}\left(T(k),{ }^{o} T\right)^{W_{d}}$, and an 
injection between $\mathbb{Q}$-vector spaces which becomes an isomorphism after tensoring with $\mathbb{C}$ must itself be an isomorphism. Hence $i \circ S: H_{\mathbb{Q}}(G(k), K) \cong H_{\mathbb{Q}}\left(T(k),{ }^{o} T\right)^{W_{d}}$ and now composing with $m_{\pi}$ the result follows easily.

Thus for groups with a twisting element, our $L$-notions and $C$-notions can be twisted into each other.

5.3. Adjoining a twisting element. If $G$ has a twisting element then we have just seen that one can, by twisting, pass between our $L$ - and $C$ - notions. What can one do when $G$ has no twisting element (for example if $G=\mathrm{PGL}_{2}$ )? In this case we will show that $G$ has a $\mathbb{G}_{m}$-extension $\widetilde{G}$ which does have a twisting element. Here we use some ideas that we learnt from reading a 2007 letter from Deligne to Serre; we thank Dick Gross for drawing our attention to this letter. We remark that previous versions of this manuscript contained a slightly messier construction involving a two-step process, reducing first via a $z$-extension to the the case where $G$ had simply-connected derived subgroup and then making another extension from there (this procedure of reducing to the case of a simply-connected derived subgroup seems to be often used in the literature but it turned out not to be necessary in this case).

Proposition 5.3.1. (a) There is a $\mathbb{G}_{m}$-extension

$$
1 \rightarrow \mathbb{G}_{m} \rightarrow \widetilde{G} \rightarrow G \rightarrow 1
$$

such that $\widetilde{G}$ has a canonical twisting element $\theta$.

(b) If $G$ has a twisting element then $\widetilde{G} \cong G \times \mathbb{G}_{m}$. More generally, there is a natural bijection between the set of splittings of $1 \rightarrow \mathbb{G}_{m} \rightarrow \widetilde{G} \rightarrow G \rightarrow 1$ and the set of twisting elements of $G$ (and in particular, the sequence splits over $F$ if and only if $G$ has a twisting element).

Proof. (a) Let $G^{\text {ad }}$ denote the quotient of $G$ by its centre and let $G^{\text {sc }}$ denote the simply-connected cover of $G^{\text {ad }}$. Over $\bar{F}$ we can choose compatible (unnamed) Borels and tori $T^{\mathrm{sc}}$ and $T^{\mathrm{ad}}$ in $G^{\mathrm{sc}}$ and $G^{\mathrm{ad}}$, and hence define the notion of a positive root in $X^{*}\left(T^{\mathrm{sc}}\right)$ and $X^{*}\left(T^{\mathrm{ad}}\right)$.

Now $G^{\text {sc }}$ is simply-connected, and hence half the sum of the positive roots for $G^{\mathrm{sc}}$ is a character $\eta$ of $T^{\mathrm{sc}}$ and hence induces a character $\gamma$ of $Z:=\operatorname{ker}\left(G^{\mathrm{sc}} \rightarrow G^{\mathrm{ad}}\right)$. This character $\gamma$ is independent of the notion of positivity because all Borels in $G \frac{\text { sc }}{F}$ are conjugate. It takes values in $\mu_{2}$ because $\eta^{2}$, the sum of the positive roots, is a character of $T^{\text {ad }}$ and is hence trivial on $Z$. Furthermore $\gamma$ is independent of the choice of $T^{\mathrm{sc}}$, and defined over $F$.

Pushing the diagram

$$
0 \rightarrow Z \rightarrow G^{\mathrm{sc}} \rightarrow G^{\mathrm{ad}} \rightarrow 0
$$

out along $\gamma: Z \rightarrow \mathbb{G}_{m}$ gives us an extension

$$
0 \rightarrow \mathbb{G}_{m} \rightarrow G^{1} \rightarrow G^{\mathrm{ad}} \rightarrow 0
$$

of groups over $F$ and now pulling back along $G \rightarrow G^{\text {ad }}$ gives us an extension

$$
0 \rightarrow \mathbb{G}_{m} \rightarrow \widetilde{G} \rightarrow G \rightarrow 0
$$

The group $\widetilde{G}$ is the extension we seek. One can define it "all in one go" as a subquotient of $G \times G^{\mathrm{sc}} \times \mathbb{G}_{m}$ : it is the elements $(g, h, k)$ such that the images of $g$ and $h$ in $G^{\text {ad }}$ are equal, modulo the image of the finite group $Z$ under the map sending $z$ to $(1, z, \gamma(z))$. 
If $T^{1}$ and $\widetilde{T}$ are maximal tori in $G^{1}$ and $\widetilde{G}$ then the character groups of these tori fit into the following Galois-equivariant commutative diagram

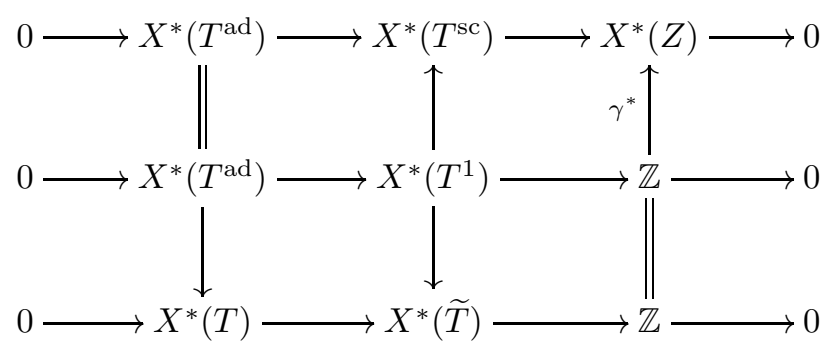

where $X^{*}\left(T^{1}\right)$ can be thought of as a pullback - the subgroup of $X^{*}\left(T^{\mathrm{sc}}\right) \oplus \mathbb{Z}$ consisting of elements whose images in $X^{*}(Z)$ coincide, and $X^{*}(\widetilde{T})$ can be thought of as a pushforward - the quotient of $X^{*}\left(T^{1}\right) \oplus X^{*}(T)$ by $X^{*}\left(T^{\text {ad }}\right)$ embedded antidiagonally.

We define $\theta$ thus: there is an element $\theta^{1}$ of $X^{*}\left(T^{1}\right)$ whose image in $X^{*}\left(T^{\mathrm{sc}}\right)$ is $\eta$, and whose image in $\mathbb{Z}$ is 1 . We let $\theta$ be the image of $\theta^{1}$ in $X^{*}(\widetilde{T})$.

We claim that $\theta$ is a twisting element for $\widetilde{G}$, and this will suffice to prove part (a). Note that the snake lemma implies that $X^{*}\left(T^{1}\right)$ injects into $X^{*}(\widetilde{T})$ and hence it suffices to show that $\theta^{1}$ is a twisting element for $G^{1}$. It is a standard fact that $\eta$ pairs to one with each simple coroot, and it follows immediately that $\theta^{1}$ pairs to one with each simple coroot. Furthermore, $\eta$ is a Galois-stable element of $X^{*}\left(T^{\mathrm{sc}}\right)$, and Galois acts trivially on $\mathbb{Z}$, from which one can deduce that Galois acts trivially on $\theta$, and so $\theta$ is indeed a twisting element.

(b) Recall that we have a short exact sequence

$$
0 \rightarrow X^{*}(T) \rightarrow X^{*}(\widetilde{T}) \rightarrow \mathbb{Z} \rightarrow 0 .
$$

If $t$ is a twisting element for $G$, then $t \in X^{*}(T)$ can be regarded as an element of $X^{*}(\widetilde{T})$ whose image in $\mathbb{Z}$ is zero. Recall that $\theta \in X^{*}(\widetilde{T})$ from (a) is a twisting element for $\widetilde{G}$, whose image in $\mathbb{Z}$ is 1 . Now consider the difference $\chi:=\theta-t$. This is an element in $X^{*}(\widetilde{T})$ which is Galois stable and pairs to zero with each simple coroot. Hence if $\widetilde{Q}$ denotes the maximal split torus quotient of $\widetilde{G}$, then $\chi$ is an element of the subgroup $X^{*}(\widetilde{Q})$ of $\widetilde{T}$. In particular, $\chi$ induces a map $\widetilde{G} \rightarrow \mathbb{G}_{m}$ defined over $F$, and the composite $\mathbb{G}_{m} \rightarrow \widetilde{G} \rightarrow \mathbb{G}_{m}$ is the identity map. It follows easily that the induced map $\widetilde{G} \rightarrow G \times \mathbb{G}_{m}$ induced by $\chi$ and the canonical map $\widetilde{G} \rightarrow G$ is an isomorphism.

This same argument shows that there is a bijection between the splitting elements for $G$ and the splittings of the short exact sequence. To give a splitting of the exact sequence is to give a map $\widetilde{G} \rightarrow \mathbb{G}_{m}$ such that the induced map $\mathbb{G}_{m} \rightarrow \widetilde{G} \rightarrow \mathbb{G}_{m}$ is the identity. If $\chi: \widetilde{G} \rightarrow \mathbb{G}_{m}$ is such a map then $\chi$ gives an element of $X^{*}(\widetilde{T})$ which is Galois stable, pairs to zero with each simple coroot, and whose image in $\mathbb{Z}$ is 1 . Conversely any such element gives a splitting. Now one checks easily that $\chi \in X^{*}(\widetilde{T})$ has these properties then $\theta-\chi(\theta$ our fixed twisting element for $\widetilde{G}$ coming from part (a)) has image zero in $\mathbb{Z}$, so can be regarded as an element of $X^{*}(T)$ that can easily be checked to be a twisting element for $G$, and conversely for any twisting element $t \in X^{*}(T)$, we have already seen that $\theta-t$ gives a splitting. 
The importance of $\widetilde{G}$ is that if $\pi$ is a $C$-algebraic representation for $G$, we can pull it back to $\widetilde{G}$ and then twist as in Proposition 5.2.2 to get an $L$-algebraic representation, for which we predict the existence of a Galois representation-but into the $L$-group of $\widetilde{G}$ rather than the $L$-group of $G$. The construction of $\widetilde{G}$ is completely canonical, and this motivates the following definition:

Definition 5.3.2. The $C$-group of $G$ is defined to be the $L$-group of $\widetilde{G}$. We will denote this group by ${ }^{C} G$.

The $C$-group is "as functorial as the $L$-group is", and naturally receives the Galois representations conjecturally (and in some cases provably) attached to $C$-algebraic automorphic representations for $G$. One can think of the $C$-group as doing, in a canonical way, the job of unravelling all the square roots that appear in the Satake isomorphism normalised a la Langlands when applied to a cohomological representation (for we shall see later on in Lemma 7.2.2 that cohomological representations are $C$-algebraic). Note that the dimension of the $C$-group is one more than the dimension of the $L$-group, but this extra degree of freedom is cancelled out by the fact that the inclusion $\mathbb{G}_{m} \rightarrow \widetilde{G}$ gives rise to a map $d:{ }^{C} G \rightarrow \mathbb{G}_{m}$ and we will see later on that our conjectures imply that if $\pi$ is $C$-algebraic for $G$ then the associated Galois representation, when composed with the map $d:{ }^{C} G\left(\overline{\mathbb{Q}}_{p}\right) \rightarrow \mathbb{G}_{m}\left(\overline{\mathbb{Q}}_{p}\right)$, is always the cyclotomic character.

It would be nice to see in a concrete manner how the $C$-group is related to the $L$-group and we achieve this in the next proposition. Let $\chi$ denote the sum of the positive roots for $G$ (after choosing some Borel and torus); then $\chi$ can be thought of as a cocharacter of a maximal torus $\widehat{T}$ of $\widehat{G}$, that is, a map $\mathbb{G}_{m} \rightarrow \widehat{T}$. Set $e=\chi(-1)$.

Proposition 5.3.3. The element $e$ is a central element of $\widehat{G}$ and is independent of all choices. There is a canonical surjection

$$
\widehat{G} \times \mathbb{G}_{m} \rightarrow \widehat{\widetilde{G}}
$$

with kernel central and of order 2 , generated by $(e,-1)$. This surjection is equivariant for the action of Galois used to define the L-groups of $G$ and $\widetilde{G}$.

Proof. We have a canonical map $c: \widetilde{G} \rightarrow G$; let us beef this up to an isogeny $\widetilde{G} \rightarrow G \times \mathbb{G}_{m}$ with kernel of order 2 . To do this we need to construct an appropriate map $\xi: \widetilde{G} \rightarrow \mathbb{G}_{m}$, which we do thus: recall that the intermediate group $G^{1}$ used in the construction of $\widetilde{G}$ was a push-out of $G^{\text {sc }} \times \mathbb{G}_{m}$ along $Z$; we define a map $G^{\text {sc }} \times \mathbb{G}_{m} \rightarrow \mathbb{G}_{m}$ by sending $(g, \lambda)$ to $\lambda^{2}$; the image of $Z$ in $\mathbb{G}_{m}$ has order at most 2, and thus this induces a map $G^{1} \rightarrow \mathbb{G}_{m}$ and hence a map $\xi: \widetilde{G} \rightarrow \mathbb{G}_{m}$. The restriction of $\xi$ to the subgroup $\mathbb{G}_{m}$ of $\widetilde{G}$ is the map $\lambda \mapsto \lambda^{2}$, and hence the kernel of the induced map $(c, \xi): \widetilde{G} \rightarrow G \times \mathbb{G}_{m}$ has order 2 and hence this map is an isogeny for dimension reasons. This isogeny induces a dual isogeny

$$
\widehat{G} \times \mathbb{G}_{m} \rightarrow \widehat{\widetilde{G}}
$$

with kernel of order 2 , and so to check that the kernel is generated by $(e,-1)$ it suffices to prove that $(e,-1)$ is in the kernel. But one easily checks that $(\chi, 1)$ maps to $2 \theta$ under the natural map

$$
X_{*}(\widehat{T}) \oplus \mathbb{Z} \rightarrow X_{*}(\widehat{\widetilde{T}})
$$


induced by the isogeny, and the result follows immediately by evaluating this cocharacter at -1 . Furthermore $(c, \xi)$ is defined over $F$ so the dual isogeny commutes with the Galois action used to define the $L$-groups.

The interested reader can use the preceding Proposition to compute examples of $C$-groups. For example the $C$-group of $\left(\mathrm{GL}_{n}\right)_{F}$ is isomorphic to the group $\left(\mathrm{GL}_{n} \times \mathrm{GL}_{1}\right)_{\overline{\mathbb{Q}}} \times \mathrm{Gal}(\bar{F} / F)$, as $e=(-1)^{n-1}$ and the surjection $\mathrm{GL}_{n} \times \mathrm{GL}_{1} \rightarrow$ $\mathrm{GL}_{n} \times \mathrm{GL}_{1}$ sending $(g, \mu)$ to $\left(g \mu^{n-1}, \mu^{2}\right)$ has kernel $(e,-1)$. As another example, the $C$-group of $\left(\mathrm{PGL}_{2}\right)_{F}$ is isomorphic to $\left(G L_{2}\right)_{\overline{\mathbb{Q}}} \times \mathrm{Gal}(\bar{F} / F)$ because the kernel of the natural map $\mathrm{SL}_{2} \times \mathrm{GL}_{1} \rightarrow \mathrm{GL}_{2}$ is $(e,-1)$. Note however that the $C$-group of $\left(\mathrm{PGL}_{n}\right)_{F}$ is not in general $\left(\mathrm{GL}_{n}\right)_{\overline{\mathbb{Q}}} \times \mathrm{Gal}(\bar{F} / F)$; its connected component is $\left(\mathrm{SL}_{n} \times \mathrm{GL}_{1}\right) /\left\langle(-1)^{n-1},-1\right\rangle$, which is isomorphic to $\mathrm{SL}_{n} \times \mathrm{GL}_{1}$ if $n$ is odd, and to a central extension of $\mathrm{GL}_{n}$ by a cyclic group of order $n / 2$ if $n$ is even.

One can use the above constructions and Conjecture 3.2 .1 to formulate a conjecture associating ${ }^{C} G$-valued Galois representations to $C$-algebraic automorphic representations ( and hence, by Lemma 7.2.2 below, to cohomological automorphic representations) for an arbitrary connected reductive group $G$ over a number field. One uses Lemma 5.1.2 to pull the $C$-algebraic representation back to a $C$ algebraic representation on $\widetilde{G}$, twists using $\theta$ and the recipe in the statement of Proposition 5.2 .2 to get an $L$-algebraic representation on $\widetilde{G}$, and then uses Conjecture 3.2 .1 on this bigger group. The map denoted $|.|^{\theta-\delta}$ in the aforementioned proposition can be checked to be the map $\widetilde{G}\left(\mathbb{A}_{F}\right) \rightarrow \mathbb{R}_{>0}$ sending $g$ to $|\xi(g)|^{1 / 2}$, with $\xi: \widetilde{G} \rightarrow \mathbb{G}_{m}$ the map defined in the proof of Proposition 5.3.3 Note finally that the map $\mathbb{G}_{m} \rightarrow \widetilde{G}$ induces a map $d:{ }^{C} G \rightarrow \mathbb{G}_{m}$.

Unravelling, and in particular using Remark 2.2.1 with the $G$ there being our $\widetilde{G}$, we see that Conjecture 3.2.1 implies the following conjecture.

Conjecture 5.3.4. If $\pi$ is $C$-algebraic, then there is a finite subset $S$ of the places of $F$, containing all infinite places, all places dividing $p$, and all places where $\pi$ is ramified, and a continuous Galois representation $\rho_{\pi}=\rho_{\pi, \iota}: \operatorname{Gal}(\bar{F} / F) \rightarrow{ }^{C} G\left(\overline{\mathbb{Q}}_{p}\right)$, which satisfies

- The composite of $\rho_{\pi}$ and the natural projection ${ }^{C} G\left(\overline{\mathbb{Q}}_{p}\right) \rightarrow \operatorname{Gal}(\bar{F} / F)$ is the identity map.

- The composite of $\rho_{\pi}$ and $d$ is the cyclotomic character.

- If $v \notin S$, then $\left.\rho_{\pi}\right|_{W_{F_{v}}}$ is $\widehat{\widetilde{G}}\left(\overline{\mathbb{Q}}_{p}\right)$-conjugate to the representation sending $w \in W_{F_{v}}$ to $\iota\left(r_{\pi_{v}}(w) \hat{\xi}\left(|w|^{1 / 2}\right)\right)$, where $\hat{\xi}$ is the map $\mathbb{C}^{\times} \rightarrow \widehat{\widetilde{G}}(\mathbb{C})$ dual to $\xi$, and where the norm on the Weil group sends a geometric Frobenius to the reciprocal of the size of the residue field.

- If $v$ is a finite place dividing $p$ then $\left.\rho_{\pi}\right|_{\mathrm{Gal}\left(\overline{F_{v}} / F_{v}\right)}$ is de Rham, and the Hodge-Tate cocharacter of this representation can be explicitly read off from $\pi$ via the recipe of Remark 5.3.5 below.

- If $v$ is a real place, let $c_{v} \in G_{F}$ denote a complex conjugation at $v$, let $\lambda_{\sigma_{v}}$ and $\lambda_{\tau_{v}} \in X^{*}(T) \otimes \mathbb{C}$ be the elements associated to $\pi_{v}$ in \$2.3, and let $\tilde{\lambda}_{\sigma_{v}}$ and $\tilde{\lambda}_{\tau_{v}}$ denote their images in $X^{*}(\widetilde{T}) \otimes \mathbb{C}$. Then $\tilde{\lambda}_{\sigma_{v}}+\frac{1}{2} \xi, \tilde{\lambda}_{\tau_{v}}+\frac{1}{2} \xi \in$ $X^{*}(\widetilde{T})=X_{*}(\widehat{\widetilde{T}})$ and $\rho_{\pi, \iota}\left(c_{v}\right)$ is $\widehat{G}\left(\overline{\mathbb{Q}}_{p}\right)$-conjugate to the element

$$
\iota\left(\alpha_{v}\right)=\iota\left(\left(\tilde{\lambda}_{\sigma_{v}}+\frac{1}{2} \xi\right)(i)\left(\tilde{\lambda}_{\tau_{v}}+\frac{1}{2} \xi\right)(i) r_{\pi_{v}}(j)\right)
$$


associated to the twist of the lift of $\pi$, as in Lemma 2.3.2.

Remark 5.3.5. The recipe for the Hodge-Tate cocharacter in the conjectures above is as follows. As in Remark 3.2.3, any $j: F \rightarrow \overline{\mathbb{Q}}$ gives rise to an infinite place $w$ of $F$ equipped with a fixed map $F_{w} \rightarrow \mathbb{C}$, which we use to identify $\mathbb{C}$ with an algebraic closure of $F_{w}$. The identity $\sigma: \mathbb{C} \rightarrow \mathbb{C}$ and the representation $\pi_{w}$ give rise to $\lambda_{\sigma}$ as in Remark 3.2.3. except that this time $\lambda_{\sigma} \in\left(X^{*}(T) \otimes_{\mathbb{Z}} \frac{1}{2} \mathbb{Z}\right) / W$. We thus obtain an element $\tilde{\lambda}_{\sigma} \in\left(X^{*}(\widetilde{T}) \otimes_{\mathbb{Z}} \frac{1}{2} \mathbb{Z}\right) / W$. Then $\tilde{\lambda}_{\sigma}+\frac{1}{2} \xi \in X^{*}(\widetilde{T}) / W$, and our conjecture is that this element $\tilde{\lambda}_{\sigma}+\frac{1}{2} \xi$ is the Hodge-Tate cocharacter associated to the embedding $F_{v} \rightarrow \overline{\mathbb{Q}}_{p}$.

We end this section by showing that the results in it imply the equivalence of Conjectures 3.1.5 and 3.1.6 (made for all groups simultaneously).

Proposition 5.3.6. Let $G$ be a connected reductive group over a number field. If Conjecture 3.1.5 is true for $\widetilde{G}$ then Conjecture [3.1.6 is true for G. Similarly if Conjecture 3.1.6 is true for $\widetilde{G}$ then Conjecture 3.1.5 is true for $G$.

Proof. We prove the first assertion; the second one is similar. Say $G$ is connected and reductive, and $\pi$ is $C$-arithmetic (resp. $C$-algebraic). Let $\pi^{\prime}$ be the pullback of $\pi$ to $\widetilde{G}$. Then $\pi^{\prime}$ is $C$-arithmetic (resp. $C$-algebraic) by Lemma 5.1.2. By Proposition 5.2 .3 (resp. Proposition 5.2.2) $\pi^{\prime} \otimes|.|^{\theta-\delta}$ is $L$-arithmetic (resp. $L$-algebraic). Applying Conjecture 3.1 .5 to $\widetilde{G}$ we deduce that $\pi^{\prime} \otimes|.|^{\theta-\delta}$ is $L$-algebraic (resp. $L$ arithmetic). Running the argument backwards now shows us that $\pi$ is $C$-algebraic (resp. $C$-arithmetic).

\section{Functoriality.}

6.1. Suppose that $G, G^{\prime}$ are two connected reductive groups over $F$, and that we have an algebraic $L$-group homomorphism

$$
r:{ }^{L} G \rightarrow{ }^{L} G^{\prime},
$$

i.e. a homomorphism of algebraic groups over $\overline{\mathbb{Q}}$ which respects the projections to $\operatorname{Gal}(\bar{F} / F)$ (recall that we are using the Galois group rather than the Weil group when forming the $L$-group). Assume that $G^{\prime}$ is quasi-split over $F$. Then we have the following weak version of Langlands' functoriality conjecture (note that we are only demanding compatibility with the local correspondence at a subset of the unramified places, and at infinity).

Conjecture 6.1.1. If $\pi$ is an automorphic representation of $G$, then there is an automorphic representation $\pi^{\prime}$ of $G^{\prime}$, called a functorial transfer of $\pi$, such that

- For all infinite places $v$, and for all finite places $v$ at which $\pi$ and $G^{\prime}$ are unramified, $r_{\pi_{v}^{\prime}}$ is $\widehat{G}^{\prime}(\mathbb{C})$-conjugate to $r \circ r_{\pi_{v}}$.

A trivial consequence of the definitions is

Lemma 6.1.2. If $\pi$ is $L$-algebraic, then any functorial transfer of $\pi$ is also $L$ algebraic.

We also have the only slightly less trivial

Lemma 6.1.3. If $\pi$ is $L$-arithmetic, then any functorial transfer of $\pi$ is also $L$ arithmetic. 
Proof. This result follows from a purely local assertion. If $v$ is a finite place where $G$ and $G^{\prime}$ are unramified and if $k$ is the completion of $F$ at $v$ then the morphism $r$ of $L$-groups induces a morphism $\widehat{G} \rightarrow \widehat{G}^{\prime}$ which commutes with the action of the Frobenius at $v$. If $T_{d}$ (resp. $T_{d}^{\prime}$ ) denotes a maximal $k$-split torus in $G / k$ (resp. $\left.G^{\prime} / k\right)$ with centralizer $T\left(\right.$ resp. $T^{\prime}$ ) then the map $\widehat{G} \rightarrow \widehat{G}^{\prime}$ induces a map $\widehat{T} \rightarrow \widehat{T}^{\prime}$ (well-defined up to restricted Weyl group actions) which commutes with the action of Frobenius, and hence maps $\widehat{T}_{d} \rightarrow \widehat{T}_{d}^{\prime}$ and $X^{*}\left(\widehat{T}_{d}^{\prime}\right)=X_{*}\left(T_{d}^{\prime}\right) \rightarrow X^{*}\left(\widehat{T}_{d}\right)=X_{*}\left(T_{d}\right)$. Now looking at the explicit definition of the Satake isomorphism in Proposition 6.7 of $\left[\right.$ Bor79] we see, after unravelling, that the map $\mathbb{Q}\left[X_{*}\left(T_{d}^{\prime}\right)\right]^{W_{d}^{\prime}} \rightarrow \mathbb{Q}\left[X_{*}\left(T_{d}\right)\right]^{W_{d}}$ induced from $X_{*}\left(T_{d}^{\prime}\right) \rightarrow X_{*}\left(T_{d}\right)$ above has the property that, after tensoring up to $\mathbb{C}$ and taking spectra, it sends the point in $\operatorname{Spec}\left(\mathbb{C}\left[X_{*}\left(T_{d}\right)\right]^{W_{d}}\right)$ corresponding to $r_{\pi_{v}}$ to the point in $\operatorname{Spec}\left(\mathbb{C}\left[X_{*}\left(T_{d}^{\prime}\right)\right]^{W_{d}^{\prime}}\right)$ corresponding to $r \circ r_{\pi_{v}}$. We now deduce that if the Satake parameter of $\pi_{v}$ is defined over a subfield $E$ of $\mathbb{C}$ then so is the Satake parameter of $\pi_{v}^{\prime}$ (because the homomorphism $\mathbb{Q}\left[X_{*}\left(T_{d}^{\prime}\right)\right]^{W_{d}^{\prime}} \rightarrow \mathbb{C}$ corresponding to $\pi_{v}^{\prime}$ factors through $\mathbb{Q}\left[X_{*}\left(T_{d}\right)\right]^{W_{d}}$ and hence through $E$ ) and the result follows.

In addition,

Proposition 6.1.4. If Conjecture 3.2.1 holds for $\pi$, then it holds for any functorial transfer of $\pi$.

Proof. With notation as above, one easily checks that $\rho_{\pi^{\prime}, \iota}:=r \circ \rho_{\pi, \iota}$ satisfies all the conditions of Conjecture 3.2.1

Note that functoriality relies on things normalised in Langlands' canonical way; the natural analogues of the results above in the $C$-algebraic and $C$-arithmetic cases are not true in general, because a morphism of algebraic groups does not send half the sum of the positive roots to half the sum of the positive roots in general.

\section{ReAlity CHECKS.}

7.1. By Proposition 2 of Lan79 any automorphic representation $\pi$ on $G$ is a subquotient of an induction $\operatorname{Ind}_{P\left(\mathbb{A}_{F}\right)}^{G\left(\mathbb{A}_{F}\right)} \sigma$, where $P$ is a parabolic subgroup of $G$ with Levi quotient $M$, and $\sigma$ is a cuspidal representation of $M$. If $\pi^{\prime}$ is another automorphic subquotient of $\operatorname{Ind}_{P\left(\mathbb{A}_{F}\right)}^{G\left(\mathbb{A}_{F}\right)} \sigma$, then $\pi_{v}$ and $\pi_{v}^{\prime}$ are equal for all but finitely many places, so $\pi$ is $C$-arithmetic (respectively $L$-arithmetic) if and only if $\pi^{\prime}$ is $C$-arithmetic (respectively $L$-arithmetic). The following lemma shows that $\pi$ is $C$-algebraic (respectively $L$-algebraic) if and only if $\pi^{\prime}$ is $C$-algebraic (respectively $L$-algebraic).

Lemma 7.1.1. Suppose that $\pi$ and $\pi^{\prime}$ are subquotients of a common induction $\operatorname{Ind}_{P\left(\mathbb{A}_{F}\right)}^{G\left(\mathbb{A}_{F}\right)} \sigma$. Then $\pi$ and $\pi^{\prime}$ have the same infinitesimal character.

Proof. This is immediate from the calculation of the infinitesimal character of an induction - see for example Proposition 8.22 of [Kna01].

Furthermore, we can check the compatibility of Conjecture 3.2 .1 for $\pi$ and $\pi^{\prime}$ (note that we cannot check the compatibility for Conjecture 3.2.2 because $\pi$ and $\pi^{\prime}$ may be ramified at different places).

Proposition 7.1.2. Suppose that $\pi$ and $\pi^{\prime}$ are subquotients of a common induction $\operatorname{Ind}_{P\left(\mathbb{A}_{F}\right)}^{G\left(\mathbb{A}_{F}\right)} \sigma$. Suppose that $\pi$ is L-algebraic. If Conjecture 3.2.1 is valid for $\pi$ then it is valid for $\pi^{\prime}$. 
Proof. Suppose that Conjecture 3.2.1 is valid for $\pi$. We wish to show that $\rho_{\pi^{\prime}, \iota}:=$ $\rho_{\pi, \iota}$ satisfies all the conditions in Conjecture 3.2.1. Since for all but finitely many places $\pi_{v}$ and $\pi_{v}^{\prime}$ are unramified and isomorphic, the first two conditions are certainly satisfied. The third condition is satisfied by Lemma 7.1.1. It remains to check that if $v$ is a real place, then (with obvious notation) $\lambda_{\sigma}(i) \lambda_{\tau}(i) r_{\pi_{v}}(j)$ and $\lambda_{\sigma}^{\prime}(i) \lambda_{\tau}^{\prime}(i) r_{\pi_{v}^{\prime}}(j)$ are $\widehat{G}(\mathbb{C})$-conjugate. As explained to us by David Vogan, it follows from the results of ABV92 (specifically from Theorem 1.24 and Proposition 6.16) that $\lambda_{\sigma}(-1) r_{\pi_{v}}(j)$ and $\lambda_{\sigma}^{\prime}(-1) r_{\pi_{v}^{\prime}}(j)$ are $\widehat{G}(\mathbb{C})$-conjugate. It is easy to check that these are $\widehat{G}(\mathbb{C})$-conjugate to $\lambda_{\sigma}(i) \lambda_{\tau}(i) r_{\pi_{v}}(j)$ and $\lambda_{\sigma}^{\prime}(i) \lambda_{\tau}^{\prime}(i) r_{\pi_{v}^{\prime}}(j)$ respectively (one conjugates by $\left.r_{\pi_{v}}\left(e^{-i \pi / 4}\right), r_{\pi_{v}^{\prime}}\left(e^{-i \pi / 4}\right)\right)$, as required.

More generally, if $\pi, \pi^{\prime}$ are nearly equivalent (that is, $\pi_{v}$ and $\pi_{v}^{\prime}$ are isomorphic for all but finitely many $v$ ), then $\pi$ is $C$-arithmetic (respectively $L$-arithmetic) if and only if $\pi^{\prime}$ is $C$-arithmetic (respectively $L$-arithmetic). We would like to be able to prove as above that $\pi$ and $\pi^{\prime}$ have the same infinitesimal character, and we would like to obtain the analogue of Proposition 7.1.2. Unfortunately, these seem in general to be beyond the reach of current techniques. However, we can prove these results for $\mathrm{GL}_{n}$, and we can then deduce them for general groups under the assumption of functoriality.

Proposition 7.1.3. If $G=\mathrm{GL}_{n}$ and $\pi, \pi^{\prime}$ are nearly equivalent, then $\pi, \pi^{\prime}$ have the same infinitesimal character. Suppose further that $\pi$ is L-algebraic. If Conjecture 3.2 .1 is valid for $\pi$ then it is valid for $\pi^{\prime}$.

Proof. By the strong multiplicity one theorem for isobaric representations (Theorem 4.4 of [JS81] $), \pi, \pi^{\prime}$ are both subquotients of a common induction $\operatorname{Ind}_{P\left(\mathbb{A}_{F}\right)}^{G\left(\mathbb{A}_{F}\right)} \sigma$. The result follows from Lemma 7.1.1 and Proposition 7.1.2.

Proposition 7.1.4. Let $G$ be arbitrary. Assume Conjecture 6.1.1. If $\pi$ and $\pi^{\prime}$ are nearly equivalent automorphic representations of $G$, then for any infinite place $v, \pi_{v}$ and $\pi_{v}^{\prime}$ have the same infinitesimal characters. Suppose further that $\pi$ is $L$-algebraic. If Conjecture 3.2.1 is valid for $\pi$ then it is valid for $\pi^{\prime}$.

Proof. For each infinite place $v$ choose an injection $\bar{F} \rightarrow \bar{F}_{v}$. This gives us a natural injection ${ }^{L} G_{v} \rightarrow{ }^{L} G$, where $G_{v}$ is the base change of $G$ to $F_{v}$. Since $\left.r_{\pi_{v}}\right|_{\mathbb{C}^{\times}}$is valued in ${ }^{L} G_{v}$, as is $c_{v}:=\lambda_{\sigma}(i) \lambda_{\tau}(i) r_{\pi_{v}}(j)$ if $v$ is real, we see that their $\widehat{G}(\mathbb{C})$-conjugacy classes in ${ }^{L} G$ are determined by their $\widehat{G}(\mathbb{C})$-conjugacy classes in ${ }^{L} G_{v}$.

Now, the $\widehat{G}(\mathbb{C})$-conjugacy classes of semisimple elements of ${ }^{L} G_{v}(\mathbb{C})$ are determined by the knowledge of the conjugacy classes of their images under all representations of ${ }^{L} G_{v}(\mathbb{C})$. To see this, note that since the formation of the $L$-group is independent of the choice of inner form, it suffices to check this in the case where $G_{v}$ is quasi-split; but the result then follows immediately from Proposition 6.7 of Bor79.

Let $r:{ }^{L} G \rightarrow \mathrm{GL}_{n} \times \operatorname{Gal}(\bar{F} / F)$ be a homomorphism of $L$-groups. Then by Conjecture 6.1.1 there are automorphic representations $\Pi, \Pi^{\prime}$ on $\mathrm{GL}_{n}$ which are functorial transfers of $\pi, \pi^{\prime}$ respectively. By Proposition 7.1.3 $\Pi$ and $\Pi^{\prime}$ have the same infinitesimal characters. Thus for each infinite place $v,\left.r_{\Pi_{v}}\right|_{\mathbb{C} \times}$ and $\left.r_{\Pi_{v}^{\prime}}\right|_{\mathbb{C} \times}$ are conjugate, i.e. $\left.r \circ r_{\pi, l}\right|_{\mathbb{C}^{\times}}$and $\left.r \circ r_{\pi^{\prime}, l}\right|_{\mathbb{C}^{\times}}$are conjugate. Since this is true for all $r$, we see that $\left.r_{\pi, \iota}\right|_{\mathbb{C}^{\times}}$and $\left.r_{\pi^{\prime}, \iota}\right|_{\mathbb{C}^{\times}}$are conjugate, whence $\pi$ and $\pi^{\prime}$ have the same infinitesimal character. 
As in the proof of Proposition 7.1.2, it remains to check that if $v$ is a real place of $F$, then $c_{v}:=\lambda_{\sigma}(i) \lambda_{\tau}(i) r_{\pi_{v}}(j)$ and $c_{v}^{\prime}:=\lambda_{\sigma}^{\prime}(i) \lambda_{\tau}^{\prime}(i) r_{\pi_{v}^{\prime}}(j)$ are $\widehat{G}(\mathbb{C})$-conjugate. By a similar argument to that used in the first half of this proof, we see that if $r:{ }^{L} G \rightarrow \mathrm{GL}_{n} \times \operatorname{Gal}(\bar{F} / F)$ is a homomorphism of $L$-groups, then $r\left(c_{v}\right)$ and $r\left(c_{v}^{\prime}\right)$ are conjugate in $\mathrm{GL}_{n}(\mathbb{C})$. Furthermore, $c_{v}$ and $c_{v}^{\prime}$ are both semisimple. Thus $c_{v}$ and $c_{v}^{\prime}$ are $\widehat{G}(\mathbb{C})$-conjugate.

7.2. Cohomological representations. Cohomological automorphic representations provide a good testing ground for our conjectures. It follows easily (see below) that any cohomological representation is $C$-algebraic, and one can often show that they are $C$-arithmetic, too (it would not surprise us if Shimura variety experts could prove they were always $C$-arithmetic with relative ease). In the case $G=\mathrm{GL}_{n}$ these arguments are due to Clozel, who also shows that for $\mathrm{GL}_{n}$ any regular $C$-algebraic representation is cohomological after possibly twisting by a quadratic character (see Lemme 3.14 of [Clo90]).

Let $v$ be an infinite place of $F$, and let $K_{v}$ be the fixed choice of a maximal compact subgroup of $G\left(F_{v}\right)$ used in the definition of automorphic forms on $G$. Let $\mathfrak{g}_{v}$ be the complexification of the Lie algebra of $G\left(F_{v}\right)$. Recall that $\pi_{v}$ may be thought of as a $\left(\mathfrak{g}_{v}, K_{v}\right)$-module, with underlying $\mathbb{C}$-vector space $V_{v}$, say.

Definition 7.2.1. We say that $\pi_{v}$ is cohomological if there is an algebraic complex representation $U$ of $G\left(F_{v}\right)$ and a non-negative integer $i$ such that

$$
H^{i}\left(\mathfrak{g}_{v}, K_{v} ; U \otimes V_{v}\right) \neq 0 .
$$

We say that $\pi$ is cohomological if $\pi_{v}$ is cohomological for all archimedean places $v$.

Lemma 7.2.2. If $\pi$ is cohomological, then it is $C$-algebraic.

Proof. By Corollary 4.2 of [BW00, if $\pi$ is cohomological then for each archimedean place $v$ there is a continuous finite-dimensional representation $U_{v}$ of $G\left(F_{v}\right)$ such that $\pi_{v}$ and $U_{v}$ have the same infinitesimal characters. The result then follows from Lemma 7.2 .3 below.

Lemma 7.2.3. If $v$ is an archimedean place of $F$ and $U$ is a continuous finite dimensional representation of $G\left(F_{v}\right)$ with infinitesimal character $\chi_{v}$, identified with an element of $X^{*}(T) \otimes_{\mathbb{Z}} \mathbb{C}$ as in section [2.3, then $\chi_{v}-\delta \in X^{*}(T)$.

Proof. This follows almost at once from the definition of the Harish-Chandra isomorphism; see for example (5.43) in Kna02.

We note that a cuspidal cohomological unitary automorphic representation $\pi$ is also $C$-arithmetic, at least when $\pi_{\infty}$ is cohomological for the trivial representation; for we can restrict scalars down to $\mathbb{Q}$ and then follow the argument in $\S 2.3$ of [BR94]. This argument presumably works in some greater generality.

\section{RELATIONSHIP WITH THEOREMS/CONJECTURES IN THE LITERATURE.}

8.1. In [Clo90], Clozel makes a number of conjectures about certain $C$-algebraic automorphic representations for $\mathrm{GL}_{n}$. We now examine the compatibility of these conjectures with those of this paper. Clozel calls an automorphic representation of $\mathrm{GL}_{n}$ algebraic if it is $C$-algebraic and isobaric; his principal reason for restricting to isobaric representations is that he wishes to use the language of Tannakian categories. 
Let $\pi=\otimes^{\prime} \pi_{v}$ be an algebraic (in Clozel's sense) representation of $\mathrm{GL}_{n}$ over $F$. Then Clozel conjectures (see conjectures 3.7 and 4.5 of [Clo90]) that

Conjecture 8.1.1. Let $\pi_{f}=\otimes_{v \nmid \infty}^{\prime} \pi_{v}$. Then there is a number field $E \subset \mathbb{C}$ such that $\pi_{f}$ is defined over $E$ (that is, such that $\pi_{f} \otimes_{\mathbb{C}, \sigma} \mathbb{C} \cong \pi_{f}$ for all automorphisms $\sigma$ of $\mathbb{C}$ which fix E pointwise). In addition, Conjecture 3.2.2 holds for $\pi \otimes|\cdot|^{(n-1) / 2}$.

(In fact, Clozel conjectures much more than this - he conjectures that there is a motive whose local $L$-factors agree with those of $\pi \otimes|\cdot|^{(n-1) / 2}$ at all finite places; the required Galois representation is then obtained as the $p$-adic realisation of this motive.)

By Proposition 7.1 .3 we see, since any automorphic representation of $\mathrm{GL}_{n}$ is nearly equivalent to an isobaric one, that Conjecture 8.1.1 implies Conjecture 3.2 .1 for $\mathrm{GL}_{n}$, and in fact an examination of the proof shows that it implies Conjecture 3.2.2. We claim that it also implies that $\pi$ is $C$-arithmetic; in fact, this follows at once from Proposition 3.1(iii) of [Clo90. Thus for $\mathrm{GL}_{n}$ our conjectures follow from those of Clozel.

The reason that our conjectures are weaker than Clozel's conjectures is that for groups other than $\mathrm{GL}_{n}$ we do not have as good an understanding of the local Langlands correspondence - for a general group $G$ we cannot even formulate a version of Conjecture 3.2 .2 which includes behaviour at the bad places without also formulating a precise local Langlands conjecture in full generality. Even for $G L_{n}$ Clozel had to be careful, restricting to isobaric representations in order not to make a conjecture which was trivially false, and such phenomena would also show up in the general case, and are typically even less well-understood here.

8.2. In Gro99 Gross presents a conjecture which assigns a Galois representation to an automorphic representation on a group $G$ with the property that any arithmetic subgroup is finite (in fact Gross gives six conditions equivalent to this in Proposition 1.4 of [Gro99]). We now discuss the relationship of this conjecture to our conjectures. Gross' assumptions imply that $G$ splits over a CM field $L$, and he also assumes that $G$ has a twisting element $\eta$ in the sense of Definition [5.2.1, In fact, Gross has informed us that one should in addition assume that the group $G$ is semisimple and simply connected, so we make this assumption from now on. This assumption in fact implies that one can take $\eta=\delta$ in the below, but for those who want to be more optimistic than Gross we have kept the two notations distinct in the below.

Let $V$ be an absolutely irreducible representation of $G$ over $\mathbb{Q}$ with trivial central character. Let $S$ be a finite set of primes of size at least 2, containing all primes at which $G$ is ramified. For each $\ell \notin S$ we let $K_{\ell}$ be a hyperspecial maximal compact subgroup of $G\left(\mathbb{Q}_{\ell}\right)$, and for each $\ell \in S$ we let $K_{\ell}$ be an Iwahori subgroup of $G\left(\mathbb{Q}_{\ell}\right)$. Let $K$ be the product of the $K_{\ell}$. Then $M(V, K)$ is the space of algebraic modular forms given by

$$
\begin{aligned}
M(V, K):=\left\{f: G\left(\mathbb{A}_{\mathbb{Q}}\right) /\left(G(\mathbb{R})_{+} \times K\right)\right. & \rightarrow V: \\
f(\gamma g) & \left.=\gamma f(g) \text { for all } \gamma \in G(\mathbb{Q}), g \in G\left(\mathbb{A}_{\mathbb{Q}}\right)\right\} .
\end{aligned}
$$

Let $H_{S}$ be the unramified Hecke algebra - the restricted tensor product of the unramified Hecke algebras $H_{\ell}$ for each $\ell \notin S$. Let $H_{K}$ be the full Hecke algebra, the tensor product of $H_{S}$ and the Iwahori Hecke algebras $H_{\ell}$ at places $\ell$ in $S$. Let 
$A$ be $H_{K} \otimes \mathbb{Q}\left[\pi_{0}(G(\mathbb{R}))\right]$. This acts on $M(V, K)$ (see section 6 of [Gro99]), and we let $N$ be a simple $A$-submodule of $M(V, K)$. We assume that $N$ gives the Steinberg character on $H_{\ell}$ for all $\ell \in S$ (see section 12 of Gro99]), and if $V$ is trivial and $\prod_{l \in S} G\left(\mathbb{Q}_{l}\right)$ is compact, we exclude the case that $N$ is trivial.

By Proposition 12.3 of Gro99, $\operatorname{End}_{A}(N)$ is a CM field, and by (7.4) of Gro99, $\pi_{0}(G(\mathbb{R}))$ acts on $N$ through a character

$$
\phi_{\infty}: \pi_{0}(G(\mathbb{R})) \rightarrow\{ \pm 1\} \subset E^{\times} .
$$

By Proposition 8.5 of [Gro99], the simple submodules of $N \otimes \mathbb{C}$ may be identified (compatibly with the actions of $H_{K}$ ) with irreducible automorphic representations $\pi=\pi_{f} \otimes \pi_{\infty}$ with $\pi_{\infty} \stackrel{\sim}{\longrightarrow} V \otimes \mathbb{C}$, and $\pi_{l}$ Steinberg for all $l \in S$. For all $l \notin S$, the unramified local Langlands correspondence (i.e. the Satake isomorphism) identifies the character of $T_{l}$ on $N$ with a homomorphism $r_{N, l}: W_{\mathbb{Q}_{l}} \rightarrow{ }^{L} G(\mathbb{C})$, and $\pi_{l}$ corresponds to this parameter under the local Langlands correspondence. Fix such a representation $\pi$.

Gross then makes the following conjecture (see Conjecture 17.2 as well as (15.3) and (16.8) of Gro99]) (note that while Gross normalises his Weil groups so that an arithmetic Frobenius element corresponds to a uniformiser, he also normalises his Satake isomorphisms so that they are constructed with arithmetic Frobenii, so the comments of Remark 3.2.5 apply):

Conjecture 8.2.1. If $p$ is a prime, and $\iota: \mathbb{C} \stackrel{\sim}{\longrightarrow} \overline{\mathbb{Q}}_{p}$, then there is a continuous Galois representation

satisfying

$$
\rho_{N, L}: \operatorname{Gal}(\overline{\mathbb{Q}} / \mathbb{Q}) \rightarrow{ }^{L} G\left(\overline{\mathbb{Q}}_{p}\right)
$$

- If $l \notin S$, then $\left.\rho_{N, \iota}\right|_{W_{\mathbb{Q}_{l}}}$ is $\widehat{G}\left(\overline{\mathbb{Q}}_{p}\right)$-conjugate to $\iota\left(r_{N, l}\right) \otimes|\cdot|^{\eta-\delta}$.

- If $s_{\infty}$ is a complex conjugation in $\operatorname{Gal}(\overline{\mathbb{Q}} / \mathbb{Q})$, then $\rho_{N, \iota}\left(s_{\infty}\right)$ is $\widehat{G}\left(\overline{\mathbb{Q}}_{p}\right)$ conjugate to $\left(\iota\left(\eta(-1) \phi_{\infty}(-1)\right), s_{\infty}\right)$.

This conjecture follows from Conjecture 3.2 .2 Indeed, the representation $\pi$ is $C$-algebraic, so by Proposition $5.2 .2 \pi \otimes|\cdot|^{\eta-\delta}$ is $L$-algebraic. Applying Conjecture 3.2 .2 gives everything in Conjecture 8.2.1 (for the description of complex conjugation, see Gro07]).

Note that Gross in fact conjectures something slightly stronger; he shows that $\pi$ is $C$-algebraic, and in fact that $\pi$ is defined over $E$, and conjectures that for any place $\lambda \mid p$ of $E$ there is a natural Galois representation $\rho_{N, \lambda}: \operatorname{Gal}(\overline{\mathbb{Q}} / \mathbb{Q}) \rightarrow{ }^{L} G\left(E_{\lambda}\right)$. As Gross has explained to us, this rationality conjecture should follow from the hypothesis that $\pi$ is Steinberg at two places, together with local-global compatibility for the Galois representations at these places.

8.3. We now discuss an example drawn from CHT08. Let $F$ be a totally real field, and let $E$ be a quadratic totally imaginary extension of $F$. Let $G$ be an $n$ dimensional unitary group over $F$ which splits over $E$, and which is compact (that is, isomorphic to $U(n))$ at all infinite places. Then the dual group of $G$ is $G L_{n}$, and if we let $\operatorname{Gal}(E / F)=\{1, c\}$, then the $L$-group of $G$ is given by

$$
{ }^{L} G=\mathrm{GL}_{n} \rtimes \operatorname{Gal}(\bar{F} / F)
$$

where $\operatorname{Gal}(\bar{F} / F)$ acts on $\mathrm{GL}_{n}$ via its projection to $\operatorname{Gal}(E / F)=\{1, c\}$, with

$$
x^{c}:=\Phi_{n} x^{-t} \Phi_{n}^{-1}
$$


where $\Phi_{n}$ is an anti-diagonal matrix with alternating entries $1,-1$. Note that $\Phi_{n}^{-1}=\Phi_{n}^{t}=(-1)^{n-1} \Phi_{n}$.

By Proposition 5.3.3, we have

$$
{ }^{C} G=\left(\left(\mathrm{GL}_{n} \times \mathbb{G}_{m}\right) /\left((-1)^{n-1},-1\right)\right) \rtimes \operatorname{Gal}(\bar{F} / F)
$$

with $\operatorname{Gal}(\bar{F} / F)$ acting by

$$
(g, \mu)^{c}=\left(\Phi_{n} g^{-t} \Phi_{n}^{-1}, \mu\right) .
$$

In Section 1 of [CHT08 there is a definition of a group $\mathcal{G}_{n}$. This group is a semidirect product $\left(\mathrm{GL}_{n} \times \mathbb{G}_{m}\right) \rtimes \operatorname{Gal}(\bar{F} / F)$, but with $\operatorname{Gal}(\bar{F} / F)$ acting by

$$
(g, \mu)^{c}=\left(\mu g^{-t}, \mu\right) \text {. }
$$

There is a morphism $j:{ }^{C} G \rightarrow \mathcal{G}_{n}$ defined thus. For $(g, \mu) \in \mathrm{GL}_{n} \times \mathbb{G}_{m}$ and $\gamma \in \operatorname{Gal}(\bar{F} / E)$ we set

$$
j((g, \mu) \times \gamma)=\left(g \mu^{1-n}, \mu^{2(1-n)}\right) \times \gamma
$$

(and note that $j\left(\left((-1)^{n-1},-1\right) \times \gamma\right)=(1 \times \gamma)$. If $\tilde{c} \in \operatorname{Gal}(\bar{F} / F)$ has image $c \in$ $\operatorname{Gal}(E / F)$ then we set

$$
j(1 \times \tilde{c})=\left(\Phi_{n},(-1)^{n-1}\right) \times \tilde{c} .
$$

It is easily checked these determine a unique homomorphism $j:{ }^{C} G \rightarrow \mathcal{G}_{n}$. For $n>1, j$ is an isogeny with kernel of order $n-1$. Since $G$ is compact at infinity, any automorphic representation $\pi$ of $G\left(\mathbb{A}_{F}\right)$ is cohomological and thus by Lemma 7.2.2 is $C$-algebraic. Conjecture 5.3.4 predicts the existence of a Galois representation

$$
\rho_{\pi}: \operatorname{Gal}(\bar{F} / F) \rightarrow{ }^{C} G\left(\overline{\mathbb{Q}}_{p}\right)
$$

with the property that $d \circ \rho_{\pi}$ is the cyclotomic character $\varepsilon$ (with $d$ as in the discussion preceding Conjecture 5.3.4). One checks easily that the composite

$$
j \circ \rho_{\pi}: \operatorname{Gal}(\bar{F} / F) \rightarrow \mathcal{G}_{n}\left(\overline{\mathbb{Q}}_{p}\right)
$$

has multiplier $\varepsilon^{1-n}$ (the multiplier of a representation into $\mathcal{G}_{n}$ is its projection onto the $\mathbb{G}_{m}$ factor).

Now, under certain mild hypotheses on $G$ and $\pi$, we note that a Galois representation $r_{\pi}^{\prime}$ satisfying the properties imposed on $j \circ r_{\pi}$ by Conjecture 5.3.4 is proved to exist in CHT08] and Tay08]. Specifically, everything except the form of complex conjugation follows from Proposition 3.4.4 of [CHT08] (although see also Theorems 4.4.2 and Theorems 4.4.3 of [CHT08] for related results on $\mathrm{GL}_{n}$ whose notation may be easier to compare to the notation used in this paper), and the form of complex conjugation follows from Theorem 4.1 of Tay08. (Note when comparing the unramified places that by definition the local Langlands correspondence $\operatorname{rec}\left(\pi_{v}\right)$ used in [CHT08] is our $r_{\pi_{v}}$.)

Conversely, the constructions of CHT08, when they apply, actually imply our Conjecture 5.3 .4 for $\pi$ (up to Frobenius semisimplification at unramified finite places). To see this, note there is a morphism $j^{\prime}: \mathcal{G}_{n} \times \mathrm{GL}_{1} \rightarrow{ }^{C} G$ such that $j^{\prime} \circ(j \times d)$ is the identity; concretely, $j^{\prime}$ is defined on the identity component by

$$
((h, \mu), \lambda) \mapsto\left(h \lambda^{(n-1) / 2}, \lambda^{1 / 2}\right)
$$

(which is well-defined independent of the choice of square root by the definition of ${ }^{C} G$ ). Then we may set $r_{\pi}=j^{\prime} \circ\left(r_{\pi}^{\prime} \times \varepsilon\right)$, which is easily checked to have the required properties. 


\section{REFERENCES}

$\left[\mathrm{ABD}^{+} 66\right]$ M. Artin, J. E. Bertin, M. Demazure, P. Gabriel, A. Grothendieck, M. Raynaud, and J.-P. Serre, Schémas en groupes. Fasc. 7: Exposés 23 à 26, Séminaire de Géométrie Algébrique de l'Institut des Hautes Études Scientifiques, vol. 1963/64, Institut des Hautes Études Scientifiques, Paris, 1965/1966. MR 0207710 (34 \#7525)

[ABV92] Jeffrey Adams, Dan Barbasch, and David A. Vogan, Jr., The Langlands classification and irreducible characters for real reductive groups, Progress in Mathematics, vol. 104, Birkhäuser Boston Inc., Boston, MA, 1992. MR MR1162533 (93j:22001)

[Art02] James Arthur, A note on the automorphic Langlands group, Canad. Math. Bull. 45 (2002), no. 4, 466-482, Dedicated to Robert V. Moody. MR MR1941222 (2004a:11120)

[Bor79] A. Borel, Automorphic L-functions, Automorphic forms, representations and $L$ functions (Proc. Sympos. Pure Math., Oregon State Univ., Corvallis, Ore., 1977), Part 2, Proc. Sympos. Pure Math., XXXIII, Amer. Math. Soc., Providence, R.I., 1979, pp. 27-61. MR MR546608 (81m:10056)

[BR94] Don Blasius and Jonathan D. Rogawski, Zeta functions of Shimura varieties, Motives (Seattle, WA, 1991), Proc. Sympos. Pure Math., vol. 55, Amer. Math. Soc., Providence, RI, 1994, pp. 525-571. MR MR1265563 (95e:11051)

[Bru03] Farrell Brumley, Maass cusp forms with quadratic integer coefficients, Int. Math. Res. Not. (2003), no. 18, 983-997. MR 1962012 (2004d:11025)

[BW00] A. Borel and N. Wallach, Continuous cohomology, discrete subgroups, and representations of reductive groups, second ed., Mathematical Surveys and Monographs, vol. 67, American Mathematical Society, Providence, RI, 2000. MR MR1721403 (2000j:22015)

[BZ77] I. N. Bernstein and A. V. Zelevinsky, Induced representations of reductive p-adic groups. I, Ann. Sci. École Norm. Sup. (4) 10 (1977), no. 4, 441-472. MR MR0579172 (58 \#28310)

[Car79] P. Cartier, Representations of p-adic groups: a survey, Automorphic forms, representations and $L$-functions (Proc. Sympos. Pure Math., Oregon State Univ., Corvallis, Ore., 1977), Part 1, Proc. Sympos. Pure Math., XXXIII, Amer. Math. Soc., Providence, R.I., 1979, pp. 111-155. MR MR546593 (81e:22029)

[CHT08] Laurent Clozel, Michael Harris, and Richard Taylor, Automorphy for some l-adic lifts of automorphic mod l Galois representations, Pub. Math. IHES 108 (2008), 1-181.

[Clo90] Laurent Clozel, Motifs et formes automorphes: applications du principe de fonctorialité, Automorphic forms, Shimura varieties, and $L$-functions, Vol. I (Ann Arbor, MI, 1988), Perspect. Math., vol. 10, Academic Press, Boston, MA, 1990, pp. 77-159. MR MR1044819 (91k:11042)

[Gro99] Benedict H. Gross, Algebraic modular forms, Israel J. Math. 113 (1999), 61-93. MR MR1729443 (2001b:11037)

[Gro07] Odd Galois representations, preprint, 2007.

[Jan03] Jens Carsten Jantzen, Representations of algebraic groups, second ed., Mathematical Surveys and Monographs, vol. 107, American Mathematical Society, Providence, RI, 2003. MR MR2015057 (2004h:20061)

[JS81] H. Jacquet and J. A. Shalika, On Euler products and the classification of automorphic forms. II, Amer. J. Math. 103 (1981), no. 4, 777-815. MR MR623137 (82m:10050b)

[Kna01] Anthony W. Knapp, Representation theory of semisimple groups, Princeton Landmarks in Mathematics, Princeton University Press, Princeton, NJ, 2001, An overview based on examples, Reprint of the 1986 original. MR MR1880691 (2002k:22011)

[Kna02] L Lie groups beyond an introduction, second ed., Progress in Mathematics, vol. 140, Birkhäuser Boston Inc., Boston, MA, 2002. MR MR1920389 (2003c:22001)

[Kot84] Robert E. Kottwitz, Stable trace formula: cuspidal tempered terms, Duke Math. J. 51 (1984), no. 3, 611-650. MR MR757954 (85m:11080)

[Lan79] R. P. Langlands, On the notion of an automorphic representation:, Automorphic forms, representations and $L$-functions (Proc. Sympos. Pure Math., Oregon State Univ., Corvallis, Ore., 1977), Part 1, Proc. Sympos. Pure Math., XXXIII, Amer. Math. Soc., Providence, R.I., 1979, pp. 203-207.

[Lan97] - Representations of abelian algebraic groups, Pacific J. Math. (1997), no. Special Issue, 231-250, Olga Taussky-Todd: in memoriam. MR MR1610871 (99b:11125) 
[Sar02] Peter Sarnak, Maass cusp forms with integer coefficients, A panorama of number theory or the view from Baker's garden (Zürich, 1999), Cambridge Univ. Press, Cambridge, 2002, pp. 121-127. MR 1975448 (2004c:11053)

[Ser79] Jean-Pierre Serre, Groupes algébriques associés aux modules de Hodge-Tate, Journées de Géométrie Algébrique de Rennes. (Rennes, 1978), Vol. III, Astérisque, vol. 65, Soc. Math. France, Paris, 1979, pp. 155-188. MR MR563476 (81j:14027)

[Spr79] T. A. Springer, Reductive groups, Automorphic forms, representations and $L$-functions (Proc. Sympos. Pure Math., Oregon State Univ., Corvallis, Ore., 1977), Part 1, Proc. Sympos. Pure Math., XXXIII, Amer. Math. Soc., Providence, R.I., 1979, pp. 3-27. MR MR546587 (80h:20062)

[Tan57] Yutaka Taniyama, L-functions of number fields and zeta functions of abelian varieties., J. Math. Soc. Japan 9 (1957), 330-366. MR 0095161 (20 \#1667)

[Tay08] Richard Taylor, Automorphy for some l-adic lifts of automorphic mod l Galois representations. II, Pub. Math. IHES 108 (2008), 183-239.

[Tit79] J. Tits, Reductive groups over local fields, Automorphic forms, representations and $L$-functions (Proc. Sympos. Pure Math., Oregon State Univ., Corvallis, Ore., 1977), Part 1, Proc. Sympos. Pure Math., XXXIII, Amer. Math. Soc., Providence, R.I., 1979, pp. 29-69. MR MR546588 (80h:20064)

[Vog93] David A. Vogan, Jr., The local Langlands conjecture, Representation theory of groups and algebras, Contemp. Math., vol. 145, Amer. Math. Soc., Providence, RI, 1993, pp. 305-379. MR MR1216197 (94e:22031)

[Wal81] Michel Waldschmidt, Transcendance et exponentielles en plusieurs variables, Invent. Math. 63 (1981), no. 1, 97-127. MR MR608530 (82k:10042)

[Wal82] Sur certains caractères du groupe des classes d'idèles d'un corps de nombres, Seminar on Number Theory, Paris 1980-81 (Paris, 1980/1981), Progr. Math., vol. 22, Birkhäuser Boston, Boston, MA, 1982, pp. 323-335. MR MR693328 (85c:11097)

[Wal85] J.-L. Waldspurger, Quelques propriétés arithmétiques de certaines formes automorphes sur GL(2), Compositio Math. 54 (1985), no. 2, 121-171. MR MR783510 (87g:11061a)

E-mail address: buzzard@ic.ac.uk

Department of Mathematics, Imperial College London

E-mail address: toby.gee@imperial.ac.uk

Department of Mathematics, Imperial College London 